\title{
Dynamics of thermal ignition of spray flames in mixing layers
}

\author{
D. Martínez-Ruiz , J. Urzay, A. L. Sánchez , A. Liñán \\ and F.A. Williams ${ }^{3}$
}

Conditions are identified under which analyses of laminar mixing layers can shed light on aspects of turbulent spray combustion. With this in mind, laminar spray-combustion models are formulated for both non-premixed and partially premixed systems. The laminar mixing layer separating a hot-air stream from a monodisperse spray carried by either an inert gas or air is investigated numerically and analytically in an effort to increase understanding of the ignition process leading to stabilization of high-speed spray combustion. The problem is formulated in an Eulerian framework, with the conservation equations written in the boundary-layer approximation and with a one-step Arrhenius model adopted for the chemistry description. The numerical integrations unveil two different types of ignition behaviour depending on the fuel availability in the reaction kernel, which in turn depends on the rates of droplet vaporization and fuel-vapour diffusion. When sufficient fuel is available near the hot boundary, as occurs when the thermochemical properties of heptane are employed for the fuel in the integrations, combustion is established through a precipitous temperature increase at a well-defined thermal-runaway location, a phenomenon that is amenable to a theoretical analysis based on activation-energy asymptotics, presented here, following earlier ideas developed in describing unsteady gaseous ignition in mixing layers. By way of contrast, when the amount of fuel vapour reaching the hot boundary is small, as is observed in the computations employing the thermochemical properties of methanol, the incipient chemical reaction gives rise to a slowly developing lean deflagration that consumes the available fuel as it propagates across the mixing layer towards the spray. The flame structure that develops downstream from the ignition point depends on the fuel considered and also on the spray carrier gas, with fuel sprays carried by air displaying either a lean deflagration bounding a region of distributed reaction or a distinct double-flame structure with a rich premixed flame on the spray side and a diffusion flame on the air side. Results are calculated for the distributions of mixture fraction and scalar dissipation rate across the mixing layer 
that reveal complexities that serve to identify differences between spray-flamelet and gaseous-flamelet problems.

\section{Introduction}

Spray flames are ubiquitous in piston engines and in the combustors of jet engines, liquid-propellant rockets, and helicopters (Harrje 1972; Lefebvre 1998). Of particular relevance for ensuring stable combustion in technological applications is the ignition dynamics of high-speed fuel sprays. Contrary to the advanced knowledge available on ignition in non-premixed gaseous flames, the ignition of fuel sprays is still not thoroughly understood, as revealed by recent studies and reviews (Li 1997; Sirignano 2010; Mastorakos 2009; Ying \& Yang 2009), which point out a number of key aspects of the problem in need of additional investigation.

Because of their key role in numerous technological applications, combustion and vaprization of fuel sprays have been the subject of many modelling efforts (see Faeth 1983; Sirignano 1983; Williams 1985; Annamalai \& Ryan 1992; Li 1997; Aggarwal 1998; Crowe, Sommerfeld \& Tsuji 1998; Jenny, Roekaerts \& Beishuizen 2013 for reviews of early work). Direct numerical integrations and large-eddy simulations of spray combustion are hindered by many complicating factors (Reveillon \& Versvich 2000; Reveillon \& Vervisch 2005; Moin \& Apte 2006; Wang \& Rutland 2007; Knudsen \& Pitsch 2010; Luo et al. 2011; Shashank 2011; Neophytou, Mastorakos \& Cant 2012), including disparate length and time scales associated with the chemistry and with the multiphase nature of the flow, which is highly turbulent in most applications.

In typical liquid-fuelled burners the fuel is injected as a high-velocity liquid jet that breaks up to form the spray. The initial heating and vaporization of the liquid fuel rely on the relatively large temperatures of the surrounding gas, which may include hot combustion products and preheated air. The heat exchange between the liquid and the gas phases is enhanced by droplet dispersion arising from the predominant turbulent motion. Chemical reaction takes place once molecular mixing between the fuel vapour and the oxidizer has occurred in mixing layers separating the spray flow from the hot-air stream. Since in most applications the injection velocities are much larger than the premixed-flame propagation velocity, combustion stabilization relies on autoignition of the fuel-oxygen mixture, with the combustion stand-off distance being controlled by the interaction of turbulent transport, droplet heating and vaporization, and gas-phase chemical reactions.

Studies of laminar mixing-layer configurations have been found to be instrumental in developing understanding of turbulent combustion (Peters 2000), including the ignition of turbulent gaseous diffusion flames (Mastorakos 2009). For the spray problem at hand, the configuration selected, shown in figure 1, involves a coflow mixing layer formed between a stream of hot air moving at velocity $U_{A}$ and a monodisperse spray moving at velocity $U_{S} \sim U_{A}$. The boundary-layer approximation will be used below to describe the resulting slender flow, which exhibits different igniting behaviours depending on the characteristics of the fuel. In this approximation, consideration of the case $U_{A}=U_{S}$ enables laminar ignition distances to be related to ignition times of unstrained spray flamelets, thereby providing quantitative information 


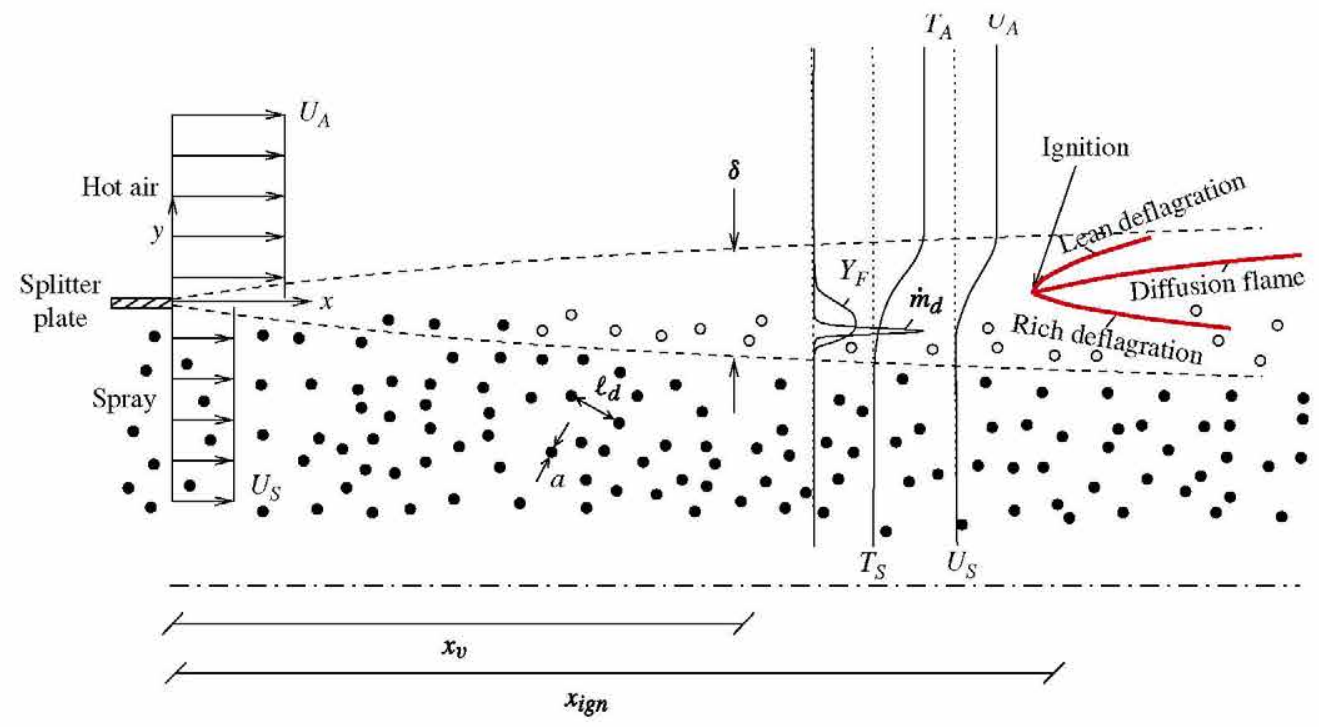

FIGURE 1. (Colour online) Sketch of the model problem: the thermal ignition of a fuel spray in a two-dimensional laminar mixing layer. Here, the black dots indicate fuel droplets, with grey droplets corresponding to vaporizing droplets. The dashed lines represent the edges of the mixing layer.

of direct applicability in regions of low scalar dissipation rate in turbulent reactive flows (see e.g. the discussion in pp. 181-186 of Peters 2000).

A two-continua formulation will be employed in the description. The gas-phase conservation equations, which include homogenized source terms associated with the force acting on and the heating and vaporization of the droplets, are accompanied by an Eulerian description of the liquid phase, with appropriate conservation equations written for the number density, velocity, temperature, and radius of the droplets in the limiting case of small values of the volume fraction occupied by the droplets. As discussed below, this Eulerian description is appropriate when the droplet size is much smaller than the inter-droplet distance, so that each droplet moves and vaporizes as an individual droplet, with negligible direct influences from neighbouring droplets. Also, the spray flow must contain many droplets, in the sense that the resulting inter-droplet distance must be much smaller than the characteristic macroscopic flow length, this last condition being required to justify a homogenized treatment of the dispersed phase.

The paper is organized as follows. Effects of droplet dispersion dynamics on ignition of sprays in turbulent mixing layers are discussed in $\$ 2$, along with the relevance and limitations of laminar-flow analyses for turbulent problems. The characteristic scales and associated dimensionless parameters for ignition in laminar mixing layers are outlined in $\S 3$, followed in $\S 4$ by the mathematical formulation of the problem, which is integrated numerically, with results presented in $\S 5$. Property values appropriate for heptane and methanol sprays in air are selected for these integrations, which will demonstrate appreciably different results. The numerical calculations include isovelocity mixing layers, yielding ignition times for unstrained spray flamelets, in some respects of greater interest in connection with turbulent flows. Section 6 is devoted to the analysis of thermal-runaway ignition, the prevailing 
ignition mode for highly volatile fuels with heptane-like properties. In $\S 7$, the mixturefraction field and associated scalar dissipation rates for spray ignition are discussed, and associated differences from purely gaseous flamelets are pointed out. Finally, concluding remarks are given in $\S 8$.

\section{Droplet dispersion and ignition in turbulent mixing layers}

Before proceeding with the analysis, it is of interest to discuss in greater detail the relevance of the laminar problem investigated below in the context of spray ignition in turbulent mixing layers. The discussion requires consideration of the transport of droplets in the presence of turbulent motion. The dynamics of the large vortices in the mixing layer is characterized by the integral time scale

$$
t_{\ell}=\ell / U,
$$

with $\ell$ being the integral length (i.e. the characteristic thickness of the turbulent mixing layer) and $U$ being related to the mean streamwise velocity. This time is to be compared with the characteristic acceleration time of the droplets. When the motion around the droplet is dominated by molecular transport, as occurs when the droplets are sufficiently small, this acceleration time (or Stokes time) is of the order of the droplet vaporization time, defined below in (3.4), which can be therefore used to define an integral-scale Stokes number

$$
S t=t_{v} / t_{\ell},
$$

the parameter controlling the overall dispersion characteristics in particle-laden turbulent mixing layers (Longmire \& Eaton 1992). Different values of St are associated with different regimes of droplet dispersion, as depicted in figure $2(a-c)$.

For $S t \gg 1$ the droplets on the spray side of the mixing layer are insensitive to the velocity perturbations induced by the large vortical motion and therefore continue in straight trajectories, as sketched in figure $2(b)$. In this slip regime, the droplets remain surrounded by the cold carrier gas, thereby hindering droplet vaporization.

An increasing interaction of the droplets with the turbulent eddies occurs as the Stokes number decreases, with droplet dispersion becoming optimal for $S t=O(1)$, when the compression strain effect acting in times of order $t_{\ell}$ enables the droplets to be ejected from the spray side through the high-strain vortex-braid regions, resulting in non-uniform droplet distributions (see figure $2 c$ ). Experimental evidence for these preferential-concentration effects has been reported in earlier work (see for instance Longmire \& Eaton 1992). In this scenario, the droplets cross the mixing layer to vaporize on the other side surrounded by hot air. Individual-droplet ignition is seldom observed for droplets in the sub-millimetre diameter range, because at the air temperatures typically found in applications the characteristic chemical time for ignition is much larger than the diffusion time around the droplet. Instead, the fuel vapour generated by droplet vaporization mixes with the surrounding air to form reactive pockets that are convected downstream. If the fuel concentration in these pockets is sufficiently high for the resulting mixture to be flammable, ignition occurs downstream, at a location such that the residence time becomes comparable to the chemical time for homogeneous ignition.

The description of ignition for $S t=O(1)$ is not readily amenable to a simple Eulerian modelling of the type used here because of the existence of crossing droplet trajectories as the droplets traverse the mixing layer through the vortex braids. These crossing trajectories have been observed, for instance, in counterflow configurations (Li 1997). Additional studies of ignition of counterflow sprays, including large inertial 


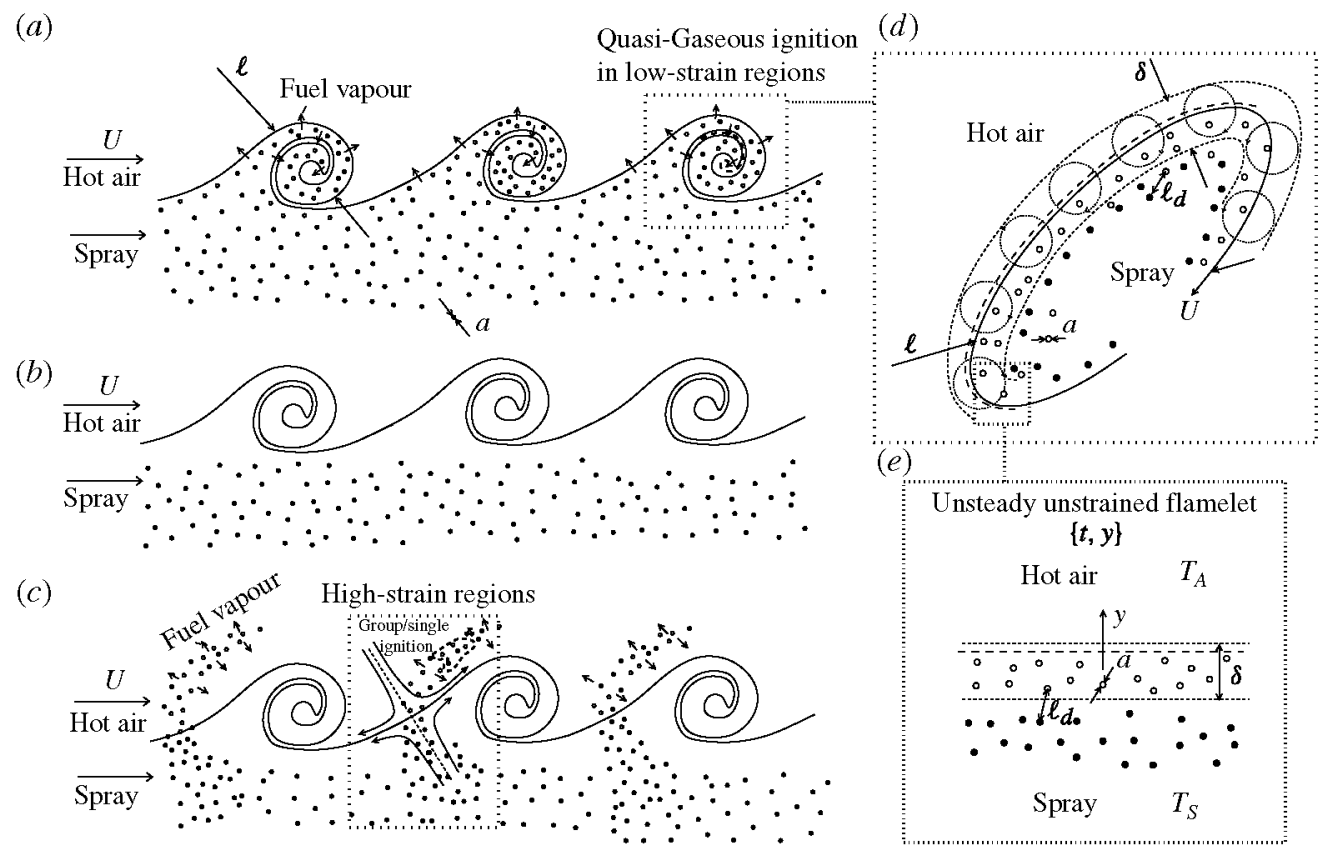

FIGURE 2. (Colour online) Spray-laden turbulent mixing layers at $(a)$ small Stokes numbers, $S t \ll 1$ (tracers regime), (b) large Stokes numbers, $S t \gg 1$ (slip regime) and (c) order-unity Stokes numbers, $S t=O(\mathbf{1})$ (preferential-concentration regime). Figure $(d)$ A sketch of the large turbulent eddies entraining the fuel spray, and $(e)$ the unsteady unstrained flamelet model of spray ignition at low Stokes numbers. Igniting regions are sketched with thickdashed lines (red colour online).

droplets crossing the stagnation plane, would clearly be beneficial in clarifying sprayignition characteristics in turbulent mixing layers for $S t=O(1)$. The present work, however, is not relevant for $S t=O(1)$ or larger, under which conditions individualdroplet combustion or droplet-cloud combustion may occur, the latter been favoured by large mass-loading ratios (Chiu \& Liu 1977; Labowsky \& Rosner 1978; Correa \& Sichel 1982).

For $S t \ll 1$, the droplets behave as flow tracers and become entrained in the largescale turbulent eddies, where they come into contact with the high-temperature air, thereby promoting vaporization and ignition of the fuel spray in the resulting mixing layers. This regime is depicted in figure $2(a)$. The strain in the vortex-braid regions promotes the transport of the fuel vapour towards the interior of the rollers, where ignition occurs more readily as a result of the existing lower strain (Wang \& Rutland 2007; Mastorakos 2009), while the larger strain rates found in the vortex-braid regions prevent ignition from occurring there by limiting fuel residence times. As suggested earlier for purely gaseous ignition (Peters 2000), the unstrained flamelet, achieved in figure 1 by setting $U_{S}$ equal to $U_{A}$, may provide an adequate representation of the ignition dynamics in the low-strain mixing regions wrapped around the core of such large vortices. As a consequence, associated ignition times, as those computed below, are relevant for quantifying ignition distances in these turbulent mixing layers.

The above discussion revolves around the effect of the large eddies associated with the integral scales of the turbulent mixing layer, which dominate the dispersion of the droplets. These large eddies coexist and interact with smaller eddies, with 
the smallest size corresponding to the Kolmogorov length scale $\ell_{k}$, which can be anticipated to be comparable with the laminar mixing-layer thickness $\delta$ in the model of figure 1, both lengths being influenced by molecular-transport effects. Although these smaller turbulent eddies may also affect mixing and reaction, their effect on ignition is less prominent than that of the large vortices, in that the cores of the large rollers correspond to regions of low strain, where ignition should occur sooner and where the unstrained laminar flamelet provides a good representation for the local flow (see figure $2 c, d$ ).

Changes to the general two-continua formulation used below would be needed in analysing turbulent flows when the Kolmogorov length scale $\ell_{k}$ attains sufficiently small values. For instance, for values of $\ell_{k}$ comparable to or smaller than the interdroplet distance, the following homogenized treatment of the droplet population is no longer a valid approximation, and rather than attempting to generalize it, a more efficient approach may be to introduce a Lagrangian description of individual droplets. For even smaller values of $\ell_{k}$, of the order of the droplet radius, unsteady effects of turbulent motion in the immediate vicinity of the droplet should be retained to calculate the forces acting on and the heating and vaporization rates of the droplets, thereby complicating significantly the description. The following development therefore precludes $\ell_{k}$ from being smaller than $\delta$, analyses with $U_{S} \neq U_{A}$ reflecting some of the aspects of influences of strain in Kolmogorov eddies.

\section{Characteristic scales}

A key parameter in describing spray dynamics is the local value of the mass-loading ratio, i.e. the ratio of the mass of liquid per unit volume to the gas density. For the configuration investigated here, the characteristic value of this quantity can be evaluated from

$$
\alpha=\frac{4}{3} \pi a_{o}^{3} n_{o} \rho_{l} / \rho_{A},
$$

in terms of the liquid-fuel density, $\rho_{l}$, the air density in the hot coflow stream, $\rho_{A}$, and the initial values of the droplet radius, $a_{o}$, and of the number of droplets per unit volume in the spray stream, $n_{o}$. In combustion applications involving liquid-fuel injection, appreciable liquid heating and vaporization resulting from heat transfer from the gas carrier occurs only downstream from the atomization region, once the droplet distribution becomes sufficiently dilute for the mass-loading ratio to decay to values of order unity. When this condition $\alpha \sim O(1)$ is used in (3.1) the relationship

$$
l_{d} \sim\left(\rho_{l} / \rho_{A}\right)^{1 / 3} a_{o} \gg a_{o}
$$

is obtained for the order of magnitude of the initial inter-droplet distance $l_{d}=n_{o}^{-1 / 3}$, with the ratio of liquid-to-gas densities $\rho_{l} / \rho_{A}$ taking values as large as

$$
\rho_{l} / \rho_{A} \sim 10^{3}
$$

for the conditions encountered in propulsion applications.

The scales $l_{d}$ and $a_{o} \ll l_{d}$ are to be compared with those of the spray-air mixing layer, associated with the acceleration, heating and vaporization of the droplets, all three processes having comparable time scales, of the order of the droplet vaporization time

$$
t_{v}=\left(\frac{\rho_{l}}{\rho_{A}}\right) \frac{a_{o}^{2}}{3 D_{T_{A}}}
$$


where $D_{T_{A}}$ denotes the air-side value of the gas thermal diffusivity. Since the chemical reaction cannot begin until after the gaseous fuel is generated, the vaporization time $t_{v}$ naturally defines the scales of the igniting mixing layer, in that ignition occurs at distances downstream from the splitter plate that are of the order of or larger than $x_{v}=U_{A} t_{v}$. At these streamwise distances, the characteristic thickness of the mixing layer is

$$
\delta \sim\left(D_{T_{A}} t_{v}\right)^{1 / 2} \sim\left(\rho_{l} / \rho_{A}\right)^{1 / 2} a_{o},
$$

which is smaller than $x_{v}$ by a factor equal to the square root of the characteristic Péclet number

$$
P e=\left(x_{v} / \delta\right)^{2}=U_{A}^{2} t_{v} / D_{T, A} .
$$

For the large values of $P e$ typically found in applications, the resulting flow is slender and correspondingly can be described in the boundary-layer approximation.

As follows from (3.2) and (3.5) with $\rho_{l} / \rho_{A} \gg 1$, the inequalities

$$
\delta \gg l_{d} \gg a_{o}
$$

can be expected to hold for mixing-layer ignition of spray flows. The disparity of length scales indicated in (3.7) enables a two-continua description of the spray ignition problem to be employed. Because of the condition $l_{d} \gg a_{o}$, each droplet vaporizes and moves as though it were isolated, surrounded by a gaseous environment created by the collective action of all of the droplets. The relatively large variations of composition and temperature that appear in the immediate vicinity of each droplet decay at distances of the order of $a_{o}$, so that in most of the gas phase between droplets the variations of the different properties are much smaller. The different gas-phase variables can be described at any spatial point by space-averaging over a neighbourhood of that point of size $d$, with $d$ in the range $\delta \gg d \gg l_{d}$. Irrespective of the inaccuracy of the most stringent condition $\left(\rho_{A} / \rho_{l}\right)^{1 / 6} \gg 1$ needed to justify $\delta \gg l_{d}$, the inequality $d \gg l_{d}$ facilitates understanding by ensuring that each averaging cell includes many droplets, so that the corresponding point sources can be homogenized, as if they were homogeneously distributed. This gives source terms that are proportional to the number of droplets per unit volume, which is the natural variable to describe in the continuum limit the droplet population of monodisperse sprays. Recent applications of this type of two-continua description include analyses of spray-jet vaporization (Arrieta-Sanagustín et al. 2011) and derivations of couplingfunction formulations for spray-flame computation (Arrieta-Sanagustín et al. 2013).

For simplicity, the chemistry describing the ignition process will be modelled with an irreversible reaction between the oxygen of the air and the fuel vapour to produce combustion products according to

$$
\mathrm{F}+s \mathrm{O}_{2} \rightarrow(1+s) \mathrm{P}+Q,
$$

where $s$ and $Q$ are, respectively, the mass of oxygen consumed and the amount of heat released per unit mass of fuel burnt. The reaction rate (mass of fuel consumed per unit volume per unit time) is assumed to be given by the Arrhenius law

$$
\omega_{F}=\rho B \hat{Y}_{O} Y_{F} \exp \left(-E_{a} / \mathbf{R}^{0} T\right)
$$

including a frequency factor $B$ and an activation energy $E_{a}$, with $\mathrm{R}^{0}$ representing the universal gas constant. In the formulation, $Y_{F}$ and $\hat{Y}_{O}=Y_{O_{2}} / Y_{O_{2 A}}$ represent the fuel-vapour and oxygen mass fractions, respectively, the latter normalized with its air-side value $Y_{O_{2 A}}$. The rate (3.9) defines a characteristic temperature-dependent 
chemical time for fuel oxidation $B^{-1} \exp \left(E_{a} / \mathrm{R}^{0} T\right)$ that can be evaluated with the air-side temperature to give the value

$$
t_{c}=B^{-1} \exp \left[E_{a} /\left(\mathbf{R}^{0} T_{A}\right)\right] .
$$

The ratio of this chemical time to the vaporization time defined in (3.4) gives the Damköhler number

$$
\Delta=t_{v} / t_{c},
$$

which enters as a prominent parameter in the problem. Autoignition data often are fitted to (3.9) to provide values of $B$ and $E_{a}$ for different fuels, but since the intention of the present work is to explore influences of spray properties rather than variations of gas-phase chemical-kinetic properties, only representative orders of magnitude of the non-dimensional gas-phase chemical-kinetic properties will be employed.

Characteristic values of $\Delta$ corresponding to realistic fuels can be evaluated by using the homogeneous ignition delay time for stoichiometric fuel-air mixtures as an estimate for $t_{c}$. For instance, for heptane at elevated pressure, the shock-wave experiments reported by Ciezki \& Adomeit (1993) give values of ignition delay times of the order of $2 \times 10^{-3} \mathrm{~s}$ at a temperature of $1000 \mathrm{~K}$. This value can be used for $t_{c}$ in evaluating (3.11) with the vaporization time $t_{v}$ obtained from (3.4) with the density of liquid heptane and with the values of the air properties at $T_{A}=1000 \mathrm{~K}$. The resulting Damköhler number, which depends strongly on the droplet size through the square of the droplet radius $a_{o}^{2}$, can be seen to become unity for $a_{o} \simeq 23 \mu \mathrm{m}$, a value of the order of those found in applications, indicating that the distinguished limit $\Delta \sim O(1)$ must be considered in addressing spray ignition. Clearly, extreme values of $\Delta$ can also be of interest, as they may appear in configurations with either higher air-side temperatures or larger droplets $(\Delta \gg 1)$ or in configurations with smaller temperatures or smaller droplet radii $(\Delta \ll 1)$.

\section{Formulation}

The vaporization time given in (3.4) will be used to define length scales for the longitudinal and transverse coordinates, $x$ and $y$, giving the dimensionless variables $x^{\prime}=x /\left(U_{A} t_{v}\right)$ and $y^{\prime}=y /\left(D_{T_{A}} t_{v}\right)^{1 / 2}$. Correspondingly, the velocity of the gas and that of the droplets will be scaled to give $u^{\prime}=u / U_{A}$ and $u_{d}^{\prime}=u_{d} / U_{A}$ for the longitudinal components and $v^{\prime}=v /\left(D_{T_{A}} / t_{v}\right)^{1 / 2}$ and $v_{d}^{\prime}=v_{d} /\left(D_{T_{A}} / t_{v}\right)^{1 / 2}$ for the transverse components.

The characteristic properties of the air stream will be used to scale the gas and droplet temperatures, $T^{\prime}=T / T_{A}$ and $T_{d}^{\prime}=T_{d} / T_{A}$, as well as the gas density, viscosity and thermal conductivity, $\rho^{\prime}=\rho / \rho_{A}, \mu^{\prime}=\mu / \mu_{A}$, and $\kappa^{\prime}=\kappa / \kappa_{A}$, respectively. Variations of the specific heat of the gas mixture will be neglected. A Fickian description will be adopted for the diffusion velocities of all species, with the binary diffusivity of species $i$ into the mixture $D_{i}^{\prime}$ scaled with its air-side value to give $D_{i}^{\prime}=D_{i} / D_{i A}$. The primes used above to denote non-dimensional variables are dropped in what follows.

A presumed power-law dependence

$$
\rho D_{i}=\mu=\kappa=T^{\sigma}
$$

is introduced for the transport properties, with $\sigma=0.7$. It is assumed that the molecular mass of the inert gas in the spray stream is close to that of air, so that prior to ignition changes in mean molecular weight of the gas mixture are only associated with the presence of fuel vapour. As a result, the equation of state can be 
written in terms of the mass fraction of fuel $Y_{F}$ in the form

$$
\rho T\left[1-Y_{F}\left(1-\frac{W_{A}}{W_{F}}\right)\right]=1,
$$

with $W_{A}$ and $W_{F}$ representing, respectively, the molecular mass of the air and the fuel.

In terms of the above dimensionless variables, the gas-phase conservation equations reduce to

$$
\begin{gathered}
\frac{\partial(\rho u)}{\partial x}+\frac{\partial(\rho v)}{\partial y}=\alpha n \dot{m}_{d}, \\
\frac{\partial(\rho u u)}{\partial x}+\frac{\partial(\rho v u)}{\partial y}=\operatorname{Pr} \frac{\partial}{\partial y}\left(T^{\sigma} \frac{\partial u}{\partial y}\right)+\alpha n \dot{m}_{d} u_{d}-\alpha n f_{x}, \\
\frac{\partial\left(\rho u Y_{F}\right)}{\partial x}+\frac{\partial\left(\rho v Y_{F}\right)}{\partial y}=\frac{1}{L e_{F}} \frac{\partial}{\partial y}\left(T^{\sigma} \frac{\partial Y_{F}}{\partial y}\right)+\alpha n \dot{m}_{d}-\Delta \Omega, \\
\frac{\partial\left(\rho u \hat{Y}_{o}\right)}{\partial x}+\frac{\partial\left(\rho v \hat{Y}_{o}\right)}{\partial y}=\frac{\partial}{\partial y}\left(T^{\sigma} \frac{\partial \hat{Y}_{o}}{\partial y}\right)-S \Delta \Omega, \\
\frac{\partial(\rho u T)}{\partial x}+\frac{\partial(\rho v T)}{\partial y}=\frac{\partial}{\partial y}\left(T^{\sigma} \frac{\partial T}{\partial y}\right)-\alpha n\left[\dot{m}_{d}\left(l_{v}-T_{d}\right)+\dot{q}_{d}\right]+q \Delta \Omega,
\end{gathered}
$$

where $\operatorname{Pr}$ represents the Prandtl number, $S=s / Y_{O_{2 A}}$ is the mass of air consumed per unit mass of fuel burnt, and $q=Q /\left(c_{p} T_{A}\right)$ and $l_{v}=L_{v} /\left(c_{p} T_{A}\right)$ are the dimensionless values of the heat of combustion (lower heating value) and latent heat of vaporization, respectively, of the fuel. While a unity Lewis number is assumed for oxygen in writing (4.6), an excellent approximation under most combustion conditions, the formulation considers a fuel-vapour Lewis number $L e_{F}$ different in general from unity, as is necessary to account for the low diffusivity of most spray fuels. The above gas-phase equations include source terms associated with the presence of the droplets in the flow, all being proportional to the number of droplets per unit volume $n$ and involving individual-droplet terms $f_{x}, f_{y}, \dot{q}_{d}$, and $\dot{m}_{d}$, to be discussed below. The dimensionless chemical reaction rate in $(4.5)-(4.7)$ is given by

$$
\Omega=\rho \hat{Y}_{O} Y_{F} \exp [\beta(T-1) / T]
$$

with $\beta=E_{a} /\left(\mathrm{R}^{0} T_{A}\right)$ denoting the non-dimensional activation energy.

The accompanying equations for the liquid phase include the conservation of droplets

$$
\frac{\partial\left(n u_{d}\right)}{\partial x}+\frac{\partial\left(n v_{d}\right)}{\partial y}=0,
$$

along with equations following their trajectories for the evolution of the droplet radius, droplet temperature, and droplet velocity

$$
\begin{aligned}
c a^{3}\left(u_{d} \frac{\partial T_{d}}{\partial x}+v_{d} \frac{\partial T_{d}}{\partial y}\right) & =\dot{q}_{d}, \\
u_{d} \frac{\partial a^{3}}{\partial x}+v_{d} \frac{\partial a^{3}}{\partial y} & =-\dot{m}_{d},
\end{aligned}
$$




$$
\begin{aligned}
& a^{3}\left(u_{d} \frac{\partial u_{d}}{\partial x}+v_{d} \frac{\partial u_{d}}{\partial y}\right)=f_{x}, \\
& a^{3}\left(u_{d} \frac{\partial v_{d}}{\partial x}+v_{d} \frac{\partial v_{d}}{\partial y}\right)=f_{y},
\end{aligned}
$$

where $c=c_{l} / c_{p}$ is the ratio of the specific heats for the two phases. These equations may be derived from the spray equation and associated conservation equations (Williams 1985), for example, by integrating over the droplet size-distribution function, which becomes a delta function for monodisperse sprays.

The values of $f_{x}, f_{y}, \dot{q}_{d}$, and $\dot{m}_{d}$ are obtained by considering the quasi-steady response of the droplet to the surrounding gaseous atmosphere, whose properties are given by the local values of the gas-phase variables at the droplet location. Additionally, although different expressions apply depending on the droplet Reynolds number associated with the relative velocity $\left(u_{d}-u, v_{d}-v\right)$, often the droplets are small enough for the near-droplet flow to be dominated by molecular transport. In that case, when the Stefan flow associated with gasification is neglected along with the variation of the viscosity with the temperature in the vicinity of the droplet, the familiar Stokes law

$$
\begin{aligned}
& f_{x}=\frac{3}{2} \operatorname{Pr} T^{\sigma} a\left(u-u_{d}\right) \\
& f_{y}=\frac{3}{2} \operatorname{Pr} T^{\sigma} a\left(v-v_{d}\right),
\end{aligned}
$$

is obtained for the force of the gas on the individual droplet.

The associated heating rate and the mass rate of vaporization follow from the analysis of the spherically symmetrical temperature field. The analysis simplifies for fuels whose latent heat of vaporization is much larger than the fuel thermal energy according to $L_{v} \gg\left(\mathrm{R}^{0} / W_{F}\right) T_{B}$, as occurs for instance for heptane $\left(L_{v} /\left(\mathrm{R}^{0} T_{B} / W_{F}\right)=11.02\right)$ and methanol $\left(L_{v} /\left(\mathrm{R}^{0} T_{B} / W_{F}\right)=12.30\right)$, because, according to the Clausius-Clapeyron relation, the mass fraction of fuel vapour at the droplet surface remains exponentially small until the droplet temperature reaches values very close to $T_{B}$. As a result, if the droplets are injected at a temperature $T_{S}<T_{B}$, there necessarily exists an initial heat-up period during which all of the heat transferred from the gas phase is employed to increase the droplet temperature from $T_{S}$ to $T_{B}$, without significant vaporization, followed by a vaporization period during which the droplet temperature remains at a value close to the boiling value. According to this simplified two-stage description, to be used below, the droplet heating and vaporization rates are to be computed according to (Liñán 1985)

$$
\dot{q}_{d}=a T^{\sigma}\left(T-T_{d}\right) \quad \text { and } \quad \dot{m}_{d}=0
$$

if $T_{d}<T_{B}$, and

$$
\dot{q}_{d}=0 \quad \text { and } \quad \dot{m}_{d}=a T^{\sigma} \ln \left(1+\frac{T-T_{B}}{l_{v}}\right)
$$

if $T_{d}=T_{B}$, with the expressions for $\dot{q}_{d}$ and $\dot{m}_{d}$ determined with the commonly used assumption of constant gas thermal conductivity around the droplet. Here, $T_{B}$ represents the boiling temperature non-dimensionalized with $T_{A}$.

The familiar Spalding expression (Godsave 1953) given in (4.17) for the vaporization rate applies to droplets vaporizing with negligible near-droplet fuel oxidation, the case of interest for spray ignition. This expression would need to be modified to describe the burning of isolated droplets (Liñán 1985; Urzay, Pitsch \& 
Liñán 2011), as may occur in some situations following ignition when the gas carrier contains oxygen and the droplet radii are sufficiently large for the droplets to sustain a surrounding flame. The possibility of ignition and burning of individual droplets is not considered further in the analysis below, which focuses on group ignition instead, the case more often encountered in applications.

Equations (4.3)-(4.7) and (4.9)-(4.13) supplemented with (4.2), (4.8), and (4.14)-(4.17), must be integrated with initial conditions at $x=0$ given by

$$
u-1=Y_{F}=\hat{Y}_{O}-1=T-1=n=0
$$

for $y>0$, and

$$
\begin{aligned}
u-u_{S} & =Y_{F}=T-T_{S}=n-1=T_{d}-T_{S}=a-1 \\
& =u_{d}-u_{S}=v_{d}=\hat{Y}_{O}-\hat{Y}_{O_{S}}=0
\end{aligned}
$$

for $y<0$, and with boundary conditions for $x>0$ given by

$$
u-1=Y_{F}=\hat{Y}_{O}-1=T-1=0
$$

as $y \rightarrow+\infty$, and

$$
u-u_{S}=Y_{F}=\hat{Y}_{O}-\hat{Y}_{O_{S}}=T-T_{S}=v=0
$$

as $y \rightarrow-\infty$, where $\hat{Y}_{O_{S}}=0$ when the spray is carried by an inert gas and $\hat{Y}_{O_{S}}=1$ when the spray is carried by air. The effect of the boundary layers developing upstream on both sides of the splitter plate has been neglected in defining the initial profiles of streamwise velocity and temperature, as is appropriate when the length of the splitter plate is much smaller than the characteristic mixing-layer length $U_{A} t_{v}$. An arbitrary condition of zero entrainment velocity on the spray side is included in writing (4.21), as applies when the mixing layer surrounds a spray jet, but this condition is inconsequential for determining the ignition distance, because of the transverse translational invariance of the problem. For $x \ll 1$, corresponding to residence times much smaller than the vaporization time, there is no appreciable coupling between the gas and liquid phases, i.e. the effect of the source terms appearing on the right-hand side of (4.3)-(4.13) is negligible in the first approximation. As a result, the initial gaseous mixing occurs according to the classical self-similar mixing-layer solution for two parallel streams (Chapman 1949; Lessen 1950), while the droplets initially maintain their velocity, temperature, and radius.

In many problems of multiphase flow, the dynamics of the dispersed phase is characterized in terms of a relevant Stokes number $S t$, defined as the ratio of the particle acceleration time to the relevant characteristic flow time. Before proceeding with the analysis, it is therefore worth discussing the role of this parameter in the context of the laminar problem considered here. For spray flow, the time scales for droplet acceleration and droplet vaporization are comparable. For instance, when the relative flow around the droplet is dominated by molecular transport, the assumption adopted in writing (4.15)-(4.17), the acceleration time (or Stokes time) is given as $2 /(3 \mathrm{Pr})$ times the vaporization time $t_{v}$. For the mixing layer, the only relevant flow time is the local residence time, so that the associated Stokes number becomes $S t=2 /(3 \operatorname{Pr} x)$ in terms of the dimensionless streamwise distance. This decaying function is such that at distances from the splitter plate of order $x_{v}$, i.e. dimensionless values $x \sim O(1)$, where ignition is anticipated to occur when $\Delta \gtrsim O(1)$, the resulting Stokes number is of order unity, so that in this region the gaseous streamlines 


$\begin{array}{lccccccc} & q & l_{v} & T_{B} & c & \frac{W_{A}}{W_{F}} & L e_{F} & S \\ \text { Heptane } & 39.5 & 0.34 & 0.37 & 2.2 & 0.29 & 2.6 & 15.2 \\ \text { Methanol } & 18.6 & 1.09 & 0.34 & 2.5 & 0.91 & 1.2 & 6.5\end{array}$

TABLE 1. Values of the dimensionless parameters used in the numerical simulations for the two liquid fuels considered.

generally differ from the droplet trajectories. For small values of the Damköhler number, however, ignition occurs at distances much larger than $x_{v}$, where the local Stokes number is very small, causing the droplet trajectories to follow closely the streamlines up to the ignition point. Nevertheless, regardless of the ignition location, the thermal expansion following the ignition of the fuel-air mixture induces large transverse velocities in a non-slender region where droplets cannot follow the gas flow, as will be seen in the plots below.

\section{Spray ignition in coflow laminar mixing layers}

The reactive spray in the laminar mixing layer was computed by numerical integration of (4.3)-(4.7) and (4.9)-(4.13), supplemented with (4.2), (4.8), and (4.14)-(4.17) and with the initial and boundary conditions given in (4.18)-(4.21). A Crank-Nicholson numerical scheme was used to integrate the parabolic gas-phase equations by marching in the $x$-direction. The liquid-phase equations were integrated by using a third-order Runge-Kutta method.

The solution depends on the thermochemical and transport properties of the fuel through the values of $q, l_{v}, T_{B}, c, S, W_{A} / W_{F}$ and $L e_{F}$, which are listed in table 1 , where the first four values are evaluated assuming $T_{A}=1000 \mathrm{~K}$ for the air-side temperature. The remaining parameters are kept fixed at representative practical values for the simulations unless mentioned otherwise, with values given by $\alpha=1, \operatorname{Pr}=0.7$, $\Delta=1, \beta=10$ and $T_{S}=T_{B}$, the latter implying that the droplets in the spray are in equilibrium with the carrier gas, where no fuel vapour is present. The integrations considered cases with $\hat{Y}_{O_{S}}=0$ and with $\hat{Y}_{O_{S}}=1$, corresponding, respectively, to sprays carried by an inert gas and by air. Although the isovelocity case $u_{S}=1.0$ is to be considered separately in $\$ 5.5$, much of the discussion below is based on computations for a spray with velocity $u_{S}=0.8$.

Sample results of the numerical integrations are shown in figure 3. In all cases, the spray mixes initially with the coflowing stream of hot air without appreciable chemical reaction. The hot-air stream provides the heat needed for droplet vaporization, which, with the scales selected, occurs over distances of order unity. The fuel vapour diffuses into the air stream, and it begins to react with the oxygen as it reaches the hightemperature boundary, located far away from the spray. Different ignition behaviours are observed in figure 3 depending on the set of parameters selected in the integrations.

\subsection{Sprays carried by an inert gas, $\hat{Y}_{O_{S}}=0$ (non-premixed systems)}

Plots of the ignition zone for sprays carried by an inert gas, shown in figure 3(a) (heptane) and figure 3(b) (methanol), display important morphological differences (independent of chemical-kinetic properties) depending on the fuel considered, with heptane ignition occurring earlier and in a more abrupt way. Differences in the 
(a)

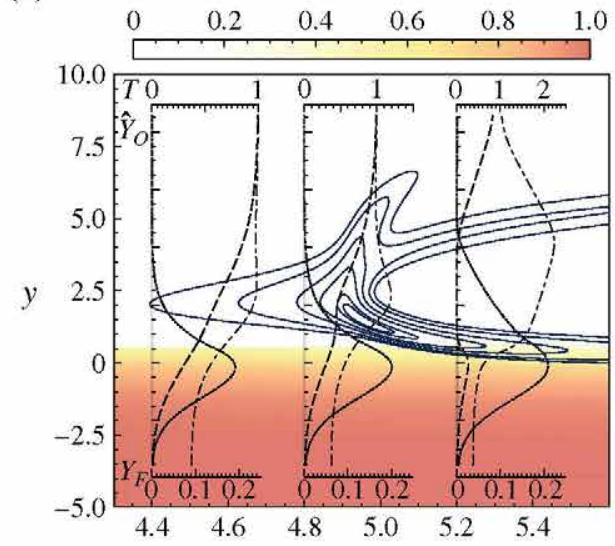

(c)

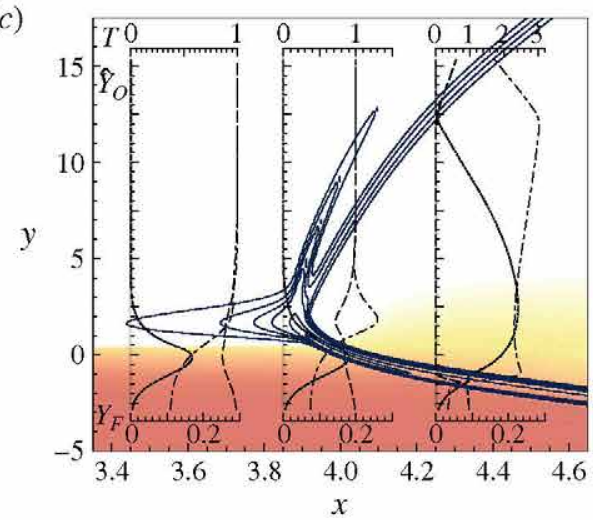

(b)

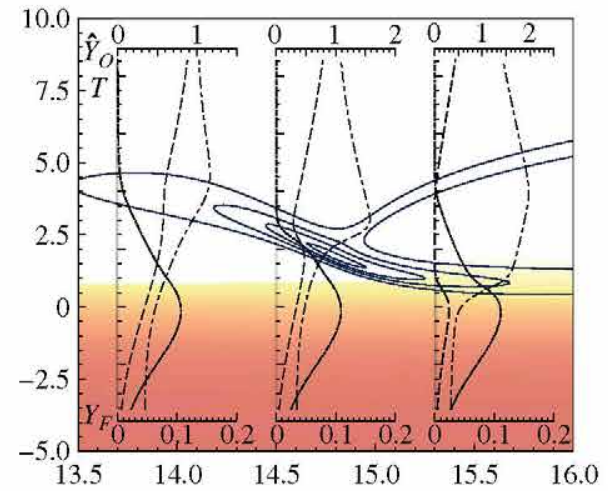

(d)

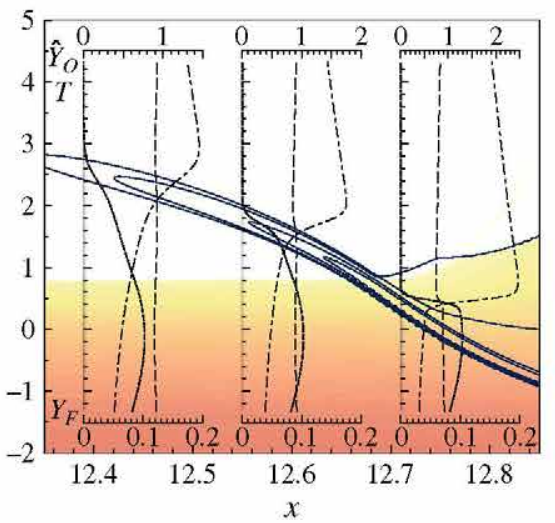

FIGURE 3. (Colour online) Ignition kernels of $(a, c)$ heptane and $(b, d)$ methanol sprays as obtained from integration of (4.3)-(4.13), with $q, l_{v}, T_{B}, c, S, W_{A} / W_{F}$ and $L e_{F}$ given in table 1 for each fuel, and $\alpha=1, T_{S}=T_{B}, u_{S}=0.8, \operatorname{Pr}=0.7, \Delta=1$, and $\beta=10$. The calculations are performed with sprays carried by $(a, b)$ inert gas and $(c, d)$ air. The figures show dimensionless reaction-rate contours (solid lines), with contour lines given by (a) $\Omega=[0.01,0.02,0.05,0.1,0.2,0.25],(b) \Omega=[0.02,0.1,0.25,0.5],(c) \Omega=$ $[0.01,0.025,0.05,0.1,0.5,2.0]$ and $(d) \Omega=[0.25,0.5,2.0,5.0]$. Shaded contours of the droplet radius $a$ are shown, with dark (red colour in online version) and white colour indicating $a=1$ and $a=0$, respectively. Temperature (dot-dashed lines), fuel mass fraction (dotted lines), and oxygen mass fraction (dashed lines) are shown in the insets for different $x$ locations.

thermochemical properties of the two fuels explain the different ignition behaviours observed. Thus, because of its smaller latent heat of vaporization $\ell_{v}$, heptane droplets tend to vaporize faster than methanol droplets. As a result, as the mixing layer develops, heptane vapour becomes available for reaction earlier than methanol vapour, thereby explaining the occurrence of ignition at smaller streamwise distances. For example, for the parametric values used in figure $3(a, b)$, the resulting ignition distance, identified by the local maximum of the reaction rate, is $x_{i g n} \simeq 4.95$ for heptane and $x_{i g n} \simeq 14.8$ for methanol.

The ignition of heptane is facilitated by its chemical heat release being more than twice that of methanol, resulting in a larger temperature increase per unit mass of 
fuel burnt that facilitates the self-acceleration of the chemical reaction rate, enabling a thermal runaway to take place. The ignition kernel develops rapidly to produce a diffusion flame surrounded on the sides by lean and rich deflagration waves that burn the excess reactants, a tribrachial structure that is clearly apparent in the reaction-rate contours of figure 3(a). Additional computations, not shown in the figure, indicate that the same type of sudden thermal-runaway event leading to a tribrachial structure characterizes the ignition of heptane when the initial spray temperature is below the boiling value. The main difference in that case is that, as a consequence of the existence of a heating stage preceding the vaporization of the droplets, the resulting ignition distance becomes larger for smaller values of $T_{S}$, so that, for example, $x_{i g n} \simeq 22$ for $T_{S}=0.28$, all other parameters being those used in figure $3(a)$.

The ignition of methanol proceeds in a more gradual form. As a result of the smaller chemical heat release of methanol, when the fuel vapour reaches the hot boundary and reacts with the oxygen of the air, the associated temperature increase is not sufficient to accelerate the chemical reaction locally to produce a thermal runaway. Instead, the fuel is seen to burn in a lean premixed flame that propagates slowly across the mixing layer into richer regions of lower initial temperature. Upon crossing stoichiometric conditions, this slow deflagration wave gives rise to a trailing diffusion flame and to a rich premixed flame, as shown in figure $3(b)$. The rich premixed flame increases the rate of spray vaporization as it burns the oxygen pocket that has diffused earlier into the spray side of the mixing layer. This rich flame eventually extinguishes at distances of order unity downstream from the ignition kernel as the oxidizer is depleted.

The two ignition modes identified here, i.e. a thermal runaway and a slow deflagration propagation, were also encountered in the analysis of ignition in gaseous mixing layers (Liñán \& Crespo 1976). In particular, the prevalence of one mode of ignition over the other was found in that case to depend only on the value of the ratio of the temperature difference between the two streams to the temperature increase associated with adiabatic combustion of the stoichiometric mixture, which emerges as the main controlling parameter in the equidiffusional case considered by Liñan \& Crespo (1976). A thermal-runaway regime, similar to that found here for heptane, occurs when this ratio takes values smaller than 1 . By contrast, when its value exceeds unity, the steep temperature gradient found at the ignition kernel prevents the self-acceleration of the chemical reaction from taking place, leading instead to the establishment of a slow lean deflagration, similar to that observed for methanol in figure $3(b)$.

The effect of fuel diffusivity was addressed in Sánchez (1997) by introducing the fuel Lewis number as a parameter in the analysis, with the non-dimensional heat of reaction assumed to be of order unity. It was found that for diffusive fuels with Lewis numbers smaller than one a thermal runaway occurs, and the amount of fuel reaching the hot boundary is so large compared with that required to sustain the thermal runaway that fuel consumption can be neglected altogether in analysing ignition, that situation applying, for example, to hydrogen (Fernández-Tarrazo, Sánchez \& Williams 2013). By contrast, when the Lewis number was larger than one, it was found (Sánchez 1997) that the amount of fuel available for reaction at the hot boundary was so small that its reaction produced a temperature increase much too small for the chemical reaction to undergo self-acceleration, and a slow deflagration emerged instead.

These observations would suggest that the behaviour seen in figure 3 would require the Lewis number of methanol to exceed that of heptane, but table 1 shows the 
opposite to be true. In spray ignition, however, the amount of fuel reaching the hot boundary depends not only on the Lewis number but also on the fuel volatility through the heat capacity and latent heat of vaporization of the liquid fuel $c$ and $l_{v}$, with the smaller values corresponding to heptane increasing the fuel supply rate. Besides, as noted previously (Liñan \& Williams 1993a), the self-acceleration of the chemical reaction is facilitated by the large value of $q$ typical of liquid fuels, so that a thermal runaway can still be the prevailing mode of mixing-layer ignition for fuels with Lewis numbers larger than unity, provided that the heat of reaction is sufficiently large. The results in figure 3 indicate that, for heptane, the combined effect of the relatively small values of $c$ and $l_{v}$ and the large value of $q$ counterbalance its low diffusivity in such a way that ignition proceeds through a thermal runaway, whereas for methanol the larger values of $c$ and $l_{v}$ and the smaller value of $q$ prevent the sudden temperature rise from taking place, with the result that a gradual ignition mode through deflagration propagation emerges instead. The role of the different parameters is to be discussed further below in connection with the theoretical analysis of the thermal-runaway regime.

\subsection{Downstream development of the diffusion flame}

The ignition-kernel plots in figure $3(a, b)$ show an emerging diffusion flame accompanied by lean and rich deflagrative waves on the sides. These deflagrations, which propagate in the mixture formed by vaporization and reactant inter-diffusion upstream from the ignition point, vanish after depleting the deficient reactants on both sides, so that in the solution encountered downstream the chemical reactions occur only in a diffusion flame burning the fuel vapour generated by the vaporizing spray with the oxygen of the air stream.

As seen in the plots, the diffusion flame emerging from the ignition kernel is very thin. This can be explained by noting that, as a result of the high temperature sensitivity of the reaction rate (3.9), the associated reaction coefficient in (4.8) becomes exponentially large as the temperature increases by chemical heat release. Consideration of the solution of (4.5)-(4.7) for large values of the effective flame Damköhler number $\Delta \exp [\beta(T-1) / T]$ leads to the well-known Burke-Schumann condition $\hat{Y}_{O} Y_{F}=0$, indicating that the reactants cannot coexist, so that the diffusion flame appears in the first approximation as a thin sheet separating a region without fuel vapour from a region without oxygen, a behaviour clearly seen in the reactant profiles of figure $3(a, b)$.

The reactants reach the flame in stoichiometric proportions by diffusion from the sides. Because of the relatively large values of $S$ corresponding to liquid fuels, the resulting non-premixed flame lies far on the air side of the mixing layer. In the solution established for $x \gg x_{i g n}$, part of the heat released at the diffusion flame is transferred by heat conduction towards the spray, where it is employed to vaporize the droplets. For $\alpha \sim O(1)$, vaporization occurs in a layer of characteristic thickness $\left(D_{T_{A}} t_{v}\right)^{1 / 2}$, corresponding to a non-dimensional thickness of order unity. Since the mixing-layer thickness increases downstream with the square root of the distance $x^{1 / 2}$, for $x \gg 1$ the vaporization layer appears as a thin front or sheath (Correa \& Sichel 1982; Arrieta-Sanagustín et al. 2011).

The above considerations indicate that the reactive spray in the mixing layer approaches, for large distances, a solution including a diffusion flame located on the air side and a thin vaporization layer at the spray edge. This structure is clearly exhibited by the profiles of temperature and reactants shown in figure 4 , obtained by extending to large values of $x$ the numerical integration of the heptane configuration 


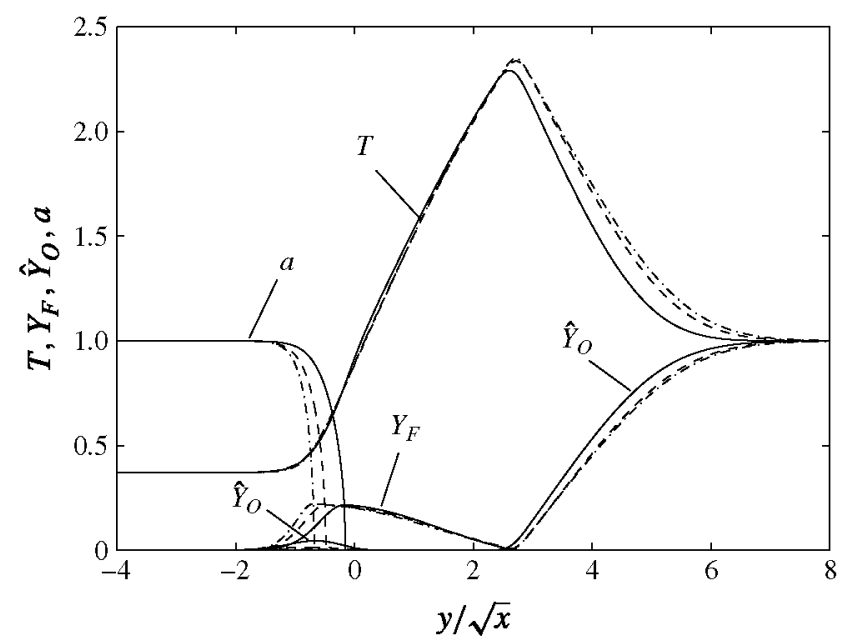

FIGURE 4. Profiles of $Y_{F}, \hat{Y}_{O}, T$, and $a$ obtained at $x=10$ (solid curves), $x=20$ (dashed curves) and $x=40$ (dot-dashed curves) from integration of (4.3)-(4.13) for the conditions of figure 3(a).

of figure 3(a). Since both the flame and the vaporization layer are much thinner than the mixing layer, the asymptotic solution in the limit $x \rightarrow \infty$ is self-similar, so that the profiles obtained numerically at different values of $x \gg 1$ collapse when expressed in terms of the rescaled similarity coordinate $y / \sqrt{x}$ employed in figure 4 . To clearly display the region where droplets are vaporizing, the plot includes the profile of droplet radius $a$, with the transition from $a=1$ to $a=0$ indicating the extent of the vaporization layer, whose relative thickness decreases with distance. The plot reveals also that the pocket of unburnt oxygen trapped on the spray side after ignition, which is still noticeable at $x=10$, is almost completely burnt at $x=20$, leaving the diffusion flame as the only reactive region across the mixing layer.

\subsection{Sprays carried by air, $\hat{Y}_{O_{S}}=1$ (partially premixed systems)}

Computations of heptane and methanol sprays carried by air were also considered. For heptane, ignition was also seen to occur in this case through a sudden temperature increase, leading to the formation of a triple flame, clearly visible in figure $3(c)$. The main difference from figure $3(a)$ pertains to the solution that emerges downstream. In figure 3(a), both premixed branches extinguish at a distance of order unity downstream from the ignition point, as the corresponding deficient reactant is depleted on each side. On the other hand, when air is employed as spray carrier, the deflagration wave developing on the rich side can propagate continuously into the spray cloud, consuming in a thin reaction layer the oxygen of the air with a fraction of the existing fuel vapour, which is generated on the spray side of the deflagration by heat conduction from the reaction region. The droplets crossing the deflagration vaporize in an oxidizer-free region, producing a large pocket of fuel vapour that has been expanded by the heat release and that diffuses to the air side to burn in a nonpremixed flame, and their trajectories now move towards the oxidizer, as can be seen by the expansion of the shaded region. This two-flame structure, resembling that observed in earlier numerical simulations of spray jet flames (Reveillon \& Vervisch 2005), is seen to persist downstream from the ignition kernel. The ultimate constant 
slope of the fuel-rich reaction zone is a measure of the premixed spray deflagration velocity.

A key ingredient for the existence of the two flames depicted in figure $3(c)$ is the relatively low value of the heat of vaporization of heptane, which facilitates the generation of a large amount of fuel vapour by droplet vaporization ahead of the deflagration, sufficient to deplete the oxygen of the spray stream, so that an intermediate oxygen-free region appears between the rich deflagration and the diffusion flame. Methanol is less volatile than heptane, and sometimes it does not develop any multiple-flame solution. For instance, in the computation of figure $3(d)$, the premixed flame originating near the hot edge of the mixing layer continues burning under lean conditions as it propagates into the spray side, because heat conduction ahead of the front can generate only a limited amount of fuel vapour, as a consequence of the relatively large heat of vaporization of methanol. The partly vaporized fuel droplets then cross the deflagration and continue to vaporize in the post-flame region, where the fuel vapour reacts with the surrounding oxidizer in a distributed manner, never establishing a diffusion flame. Under these conditions the structure of the solution downstream from the ignition point is very sensitive to the specific set of boundary conditions, so that, depending on the values selected, both heptane and methanol can, in principle, support either a single lean deflagration followed by a region of distributed reaction or, ultimately, a double-flame structure with an intermediate oxygen-free region. These calculated behaviours apply for the representative reaction-rate parameters $\Delta=1$ and $\beta=10$ selected here, while different structures could arise if values of these and other parameters were very different, thus further emphasizing the remarkable variations that may occur, especially in partially premixed spray combustion.

\subsection{Interaction of the spray cloud and the deflagration}

In understanding the leakage of the droplets across the deflagration propagating into the spray in figure $3(c, d)$ one should keep in mind that the characteristic residence time across the flame can be expected to be proportional to (although somewhat larger than) the chemical-reaction time associated with the peak flame temperature, reached at the reaction layer. This chemical time for the deflagration is much smaller than the characteristic chemical time for ignition $t_{c}$, defined in (3.10), because the latter is based on the air-side temperature of the mixing layer, which is significantly smaller than the peak flame temperature, as can be seen in the profiles of figure $3(c, d)$. As a consequence, with the Damköhler number $\Delta=t_{v} / t_{c}$ assumed to be of order unity, the resulting residence time across the flame is smaller than the characteristic vaporization time, so that only a relatively small fraction of the liquid fuel vaporizes as the droplets cross the flame. Most of the droplet vaporization occurs instead either upstream in the mixing layer or in the post-flame region, resulting in the distribution of droplet radii shown in the figures. As previously discussed, depending on the value of $\ell_{v}$ the associated deflagration can be either rich, as occurs for heptane in figure $3(c)$ or lean, as occurs for methanol in figure $3(d)$.

To better display the characteristic features of the spray/deflagration interaction, a detailed view of the solution near the front corresponding to the flow conditions of figure $3(d)$ is given in figure 5. As the mixing layer develops upstream from the ignition region, the droplets vaporize partially through the heat flux coming from the hot-air stream, creating a mixture that, for methanol droplets, is lean everywhere. This is illustrated in figure 5 by plotting the contours of the gaseous equivalence ratio $\phi_{g}=S Y_{F} / \hat{Y}_{O}$. The fuel accumulates towards the middle of the mixing layer, where a 


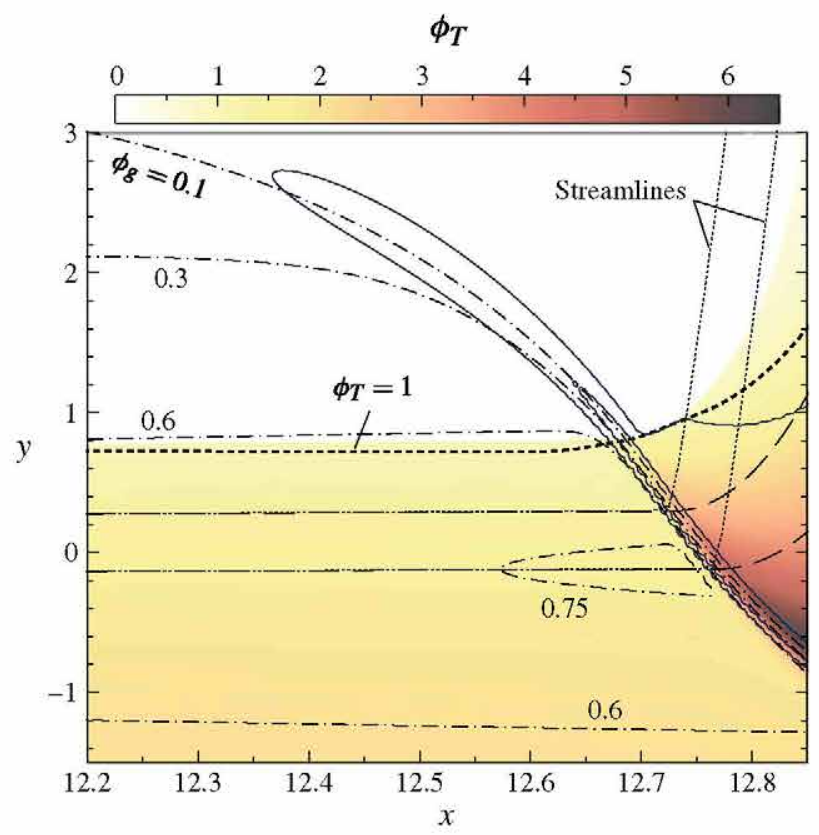

FIGURE 5. (Colour online) Close-up view of the spray-side deflagration wave of figure $3(d)$, including droplet trajectories (long-dashed lines), gaseous streamlines (dotted lines), reactionrate contours $\Omega=0.1$ and $\Omega=5$ (solid lines), contours of the gaseous equivalence ratio $\phi_{g}$ (dot-dashed lines), and a graded shade to indicate levels of global equivalence ratio $\phi_{T}$ (colour online).

maximum value $\phi_{g} \simeq 0.76$ is achieved. At that intermediate location, the transverse propagation velocity of the deflagration peaks, as indicated by the existence of an inflection point in the curved flame front. Because of the lean conditions, all of the fuel vapour available is consumed across the flame front. The droplets keep vaporizing as they cross the deflagration, and the fuel vapour generated by these droplets burns in a distributed manner in the high-temperature post-flame region with the excess of oxygen that has leaked through the front.

Besides the standard gaseous-fuel equivalence ratio $\phi_{g}=S Y_{F} / \hat{Y}_{O}$, in studying spray flames, it is convenient to introduce the liquid-based equivalence ratio $\phi_{\ell}=S \alpha n a^{3} /\left(\rho \hat{Y}_{O}\right)$. The resulting global equivalence ratio $\phi_{T}=\phi_{\ell}+\phi_{g}$, accounting for all of the fuel present in the flow, is represented by use of a graded shade in the plot as well as a thick dotted line indicating the region where $\phi_{T}=1$. The initial value of $\phi_{T}$ in the fuel stream was $\phi_{T}=2.21$ in these calculations, while it was $\phi_{T}=5.17$ for the heptane spray in figure $3(c)$. In the region where $\phi_{T}<1$ the local gaseous mixture is unconditionally lean, in that, even if all the liquid fuel were instantaneously vaporized, the resulting gas mixture would still be lean. The sharp increase in $\phi_{T}$ observed in the post-flame region is a result of the thermal expansion across the flame, which reduces drastically the density, inducing accompanying large transverse velocities and associated streamlines deflecting sharply at the flame front. The local mass-loading ratio increases, because the droplets cannot follow the gaseous flow, causing droplet trajectories to depart from streamlines as the flow crosses the deflagration, as shown in figure 5 . 


\subsection{Ignition of unstrained spray flamelets}

The solution for the mixing layer simplifies when the two coflowing streams have equal velocities, in which case, integration of (4.4) and (4.12) provides the uniform distribution $u=u_{d}=1$ for the streamwise velocity components of the gas and liquid phases everywhere in the flow field, a simplification that can be incorporated when writing the remaining equations (4.3), (4.5)-(4.7), (4.9)-(4.11) and (4.13). Besides describing the evolution with $x$ of the isovelocity coflow mixing layer, the resulting mathematical problem provides the temporal evolution of the mixing layer formed by putting into contact at a given time a semi-infinite space of hot air with a semi-infinite spray suspension, with $x$ being equivalent in that case to the dimensionless time $t$ obtained with use made of the characteristic vaporization time $t_{v}$. The problem formulated here thus provides the solution for ignition of unsteady, unstrained spray flamelets, indicated earlier to have been considered relevant for studies of turbulent spray ignition for low Stokes numbers.

Sample results of the numerical integrations including both an inert gas and air as spray carriers are shown in figure 6 for heptane and in figure 7 for methanol. The values of the different parameters are those employed earlier for the integrations shown in figure 3. Profiles of temperature and fuel and oxygen mass fractions are represented at four different instants of time, which are selected to illustrate the ignition behaviour. Corresponding curves from the problems whose solutions are shown in figure 3 would not be greatly different if $x$ there were replaced by $t$.

Observation of the evolution of the temperature profiles in figure 6 reveals a sudden temperature increase at a given location, corresponding to a thermal-runaway mode of ignition characteristic of heptane, while the temperature profiles in figure 7 for methanol correspond to a deflagration front progressing from the hot boundary across the mixing layer. When the spray carrier is inert, the case shown in figure $6(a-c)$ for heptane and in figure $7(a-c)$ for methanol, the solution at the last instant considered includes a central region free from oxygen bounded by a diffusion flame on the air side and a rich deflagration on the spray side, the latter front disappearing subsequently following the depletion of the oxygen pocket found on the left-hand side of figures $6(c)$ and $7(c)$.

When air is present in the spray stream, the solution at the last instant of time considered is also similar for heptane and methanol. In this isovelocity case, both fuels develop downstream the double-flame solution previously observed in figure 3(c) for heptane. Observation of the evolution of the profiles in figure $7(d-e)$ indicates that the deflagration, initially lean, becomes rich at a given instant, leading to the emergence of a large intermediate fuel pocket that burns on the air side in an emerging diffusion flame. The dramatic change in the flow structure for methanol is apparent by comparing the profiles at $t=15.5$ and $t=16.0$, which would correspond to proceeding to values of $x$ greater than 12.8 in figure $3(d)$.

\section{The thermal-runaway mode of spray ignition}

Let us now focus attention on the type of autoignition process that occurs in figure $3(a, c)$, corresponding to figure 6. During this type of spray ignition by a hot coflow the chemical reaction occurs initially near the hot boundary, where the fuel mass fraction is a small quantity of order $Y_{F_{R}} \ll 1$. It can be expected from (4.8) that because of the large activation energy the chemical reaction rate increases by a factor of order unity when the heat release causes the temperature to increase by a small relative amount, of order $\beta^{-1}$, giving rise to a self-accelerating reacting process that 


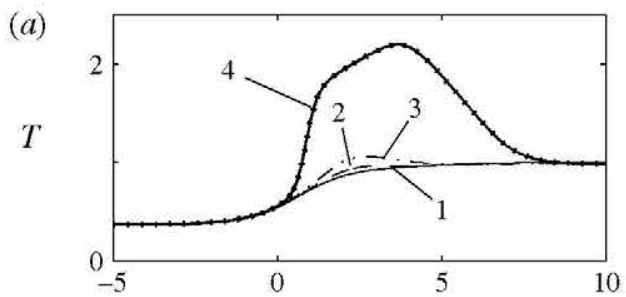

(d)
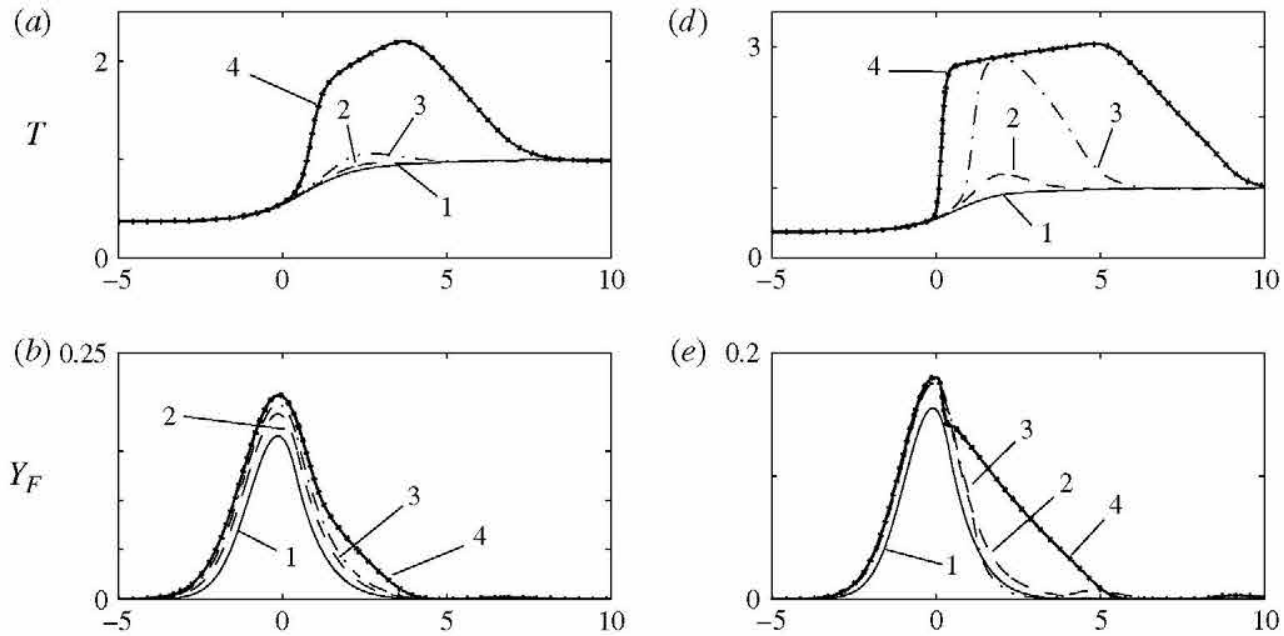

(e) 0
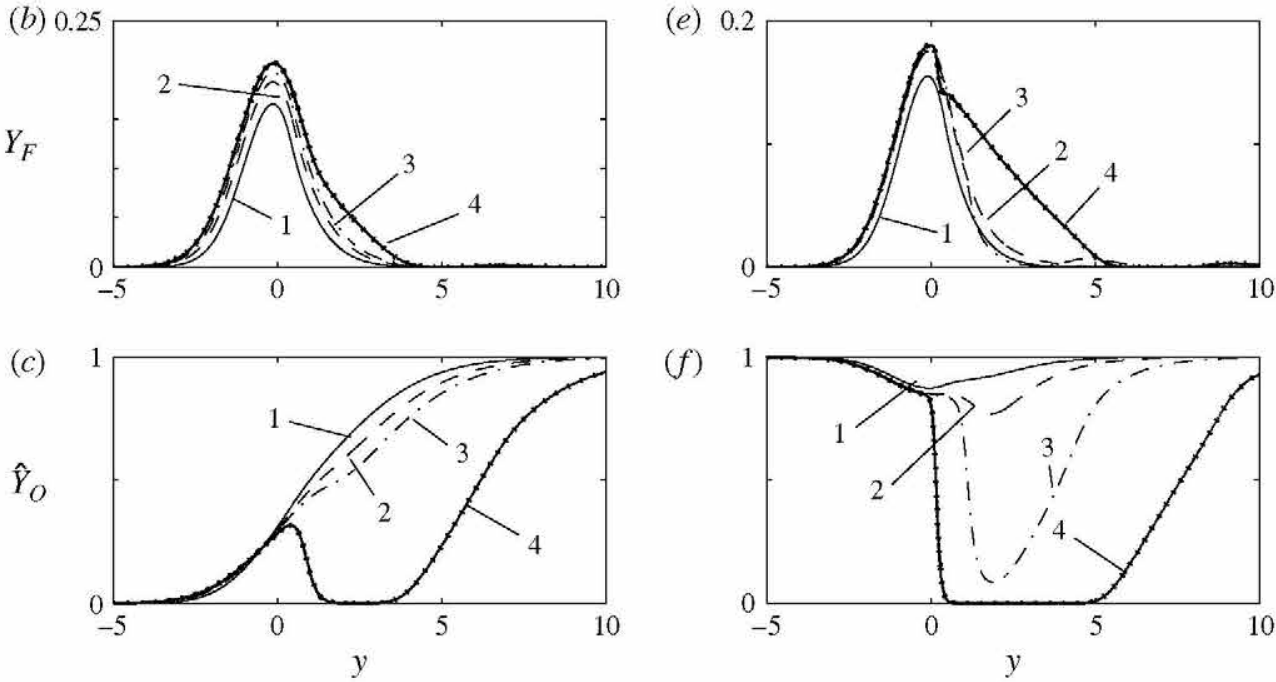

(f)

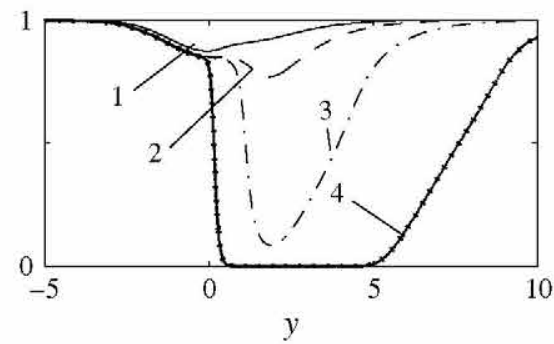

FIGURE 6. Temporal evolution of temperature, fuel, and oxidizer mass fractions in a mixing layer of air and heptane spray carried by $(a-c)$ inert gas and by $(d-f)$ air. The time instants 1 , 2,3 and 4 correspond, respectively, to $t=4.5,5.5,6.0$ and 6.5 for $(a-c)$, and to $t=4.0,4.8$, 4.9 and 5.0 for $(d-f)$.

may lead to a local thermal runaway if sufficient fuel is available. It can be concluded by comparing the reaction terms in (4.5) and (4.7) that a non-dimensional temperature increase of order $\beta^{-1}$ is associated with fuel consumption that decreases the fuel mass fraction by an amount of order $(q \beta)^{-1}$. Therefore, a sustained self-acceleration of the chemical reaction is possible only when

$$
Y_{F_{R}} \gg(q \beta)^{-1} \text {. }
$$

We examine below whether this condition is satisfied and formulate the associated ignition problem.

Because of the large value of the activation energy, during the weakly reactive stage prior to ignition, the temperature and reactant profiles are in the first approximation those resulting from the chemically frozen interaction of the spray with the hot air, to be determined by numerical integration of (4.3)-(4.13) with $\Delta=0$. The solution at distances $y \gg 1$ takes a simplified form that can be described by noticing that the streamwise velocity and density there approach their boundary values $u=\rho=1$, so that the continuity equation (4.3) reduces to $\partial v / \partial y=0$, indicating that the transverse velocity is given simply by the entrainment distribution $v=v_{\infty}(x)$. The temperature variations from the boundary value $T=1$ are obtained by integrating the energy 

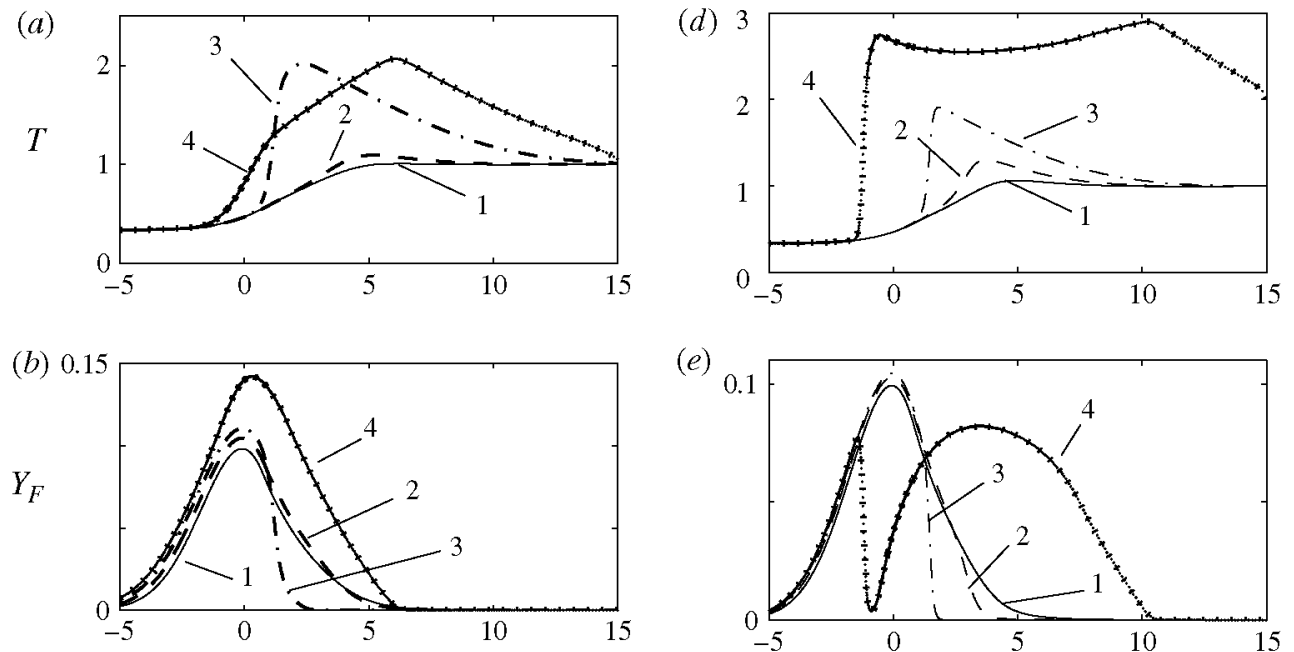

(e)
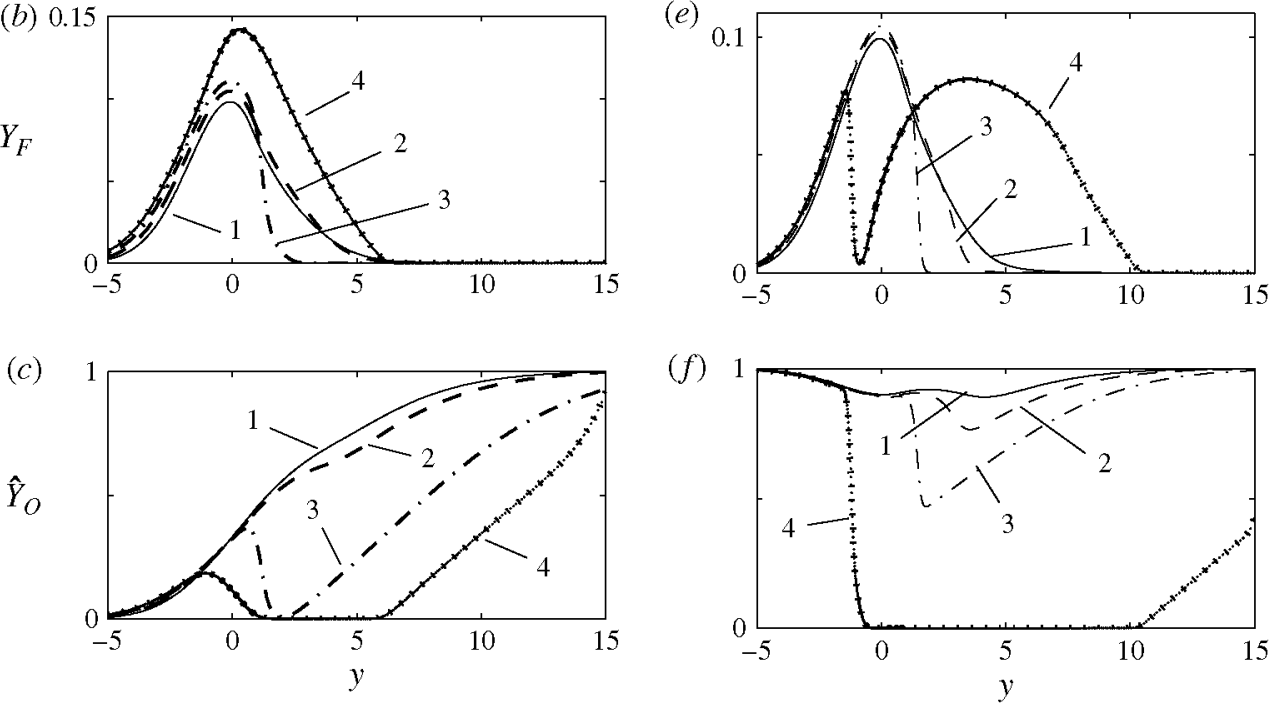

FIGURE 7. Temporal evolution of temperature, fuel, and oxidizer mass fractions in a mixing layer of air and methanol spray carried by $(a-c)$ inert gas and by $(d-f)$ air. The time instants 1 , 2,3 and 4 correspond, respectively, to $t=14.0,16.0,18.0$ and 20.0 for $(a-c)$, and to $t=14.0$, $15.0,15.5$ and 16.0 for $(d-f)$.

equation (4.7) written in the form

$$
\frac{\partial T_{f}}{\partial x}+v_{\infty} \frac{\partial T_{f}}{\partial y}=\frac{\partial^{2} T_{f}}{\partial y^{2}}
$$

for the chemically frozen temperature $T_{f}$, which further reduces to the heat equation by introduction of the alternative transverse coordinate $y+y_{T}-\int_{0}^{x} v_{\infty} \mathrm{d} x$ involving an unknown translation $y_{T}$ along with the displacement $-\int_{0}^{x} v_{\infty} \mathrm{d} x$ associated with the amount of gas entrained by the mixing layer. For $y \gg 1$, the equation admits a self-similar description in terms of the similarity diffusion variable obtained by scaling the alternative transverse coordinate with $\left(x-x_{T}\right)^{1 / 2}$, where $x_{T}$ is an appropriate virtual origin. The solution to the heat equation then leads to the well-known representation

$$
1-T_{f}=A_{T} \operatorname{erfc}\left(\frac{y+y_{T}-\int_{0}^{x} v_{\infty} \mathrm{d} x}{2\left(x-x_{T}\right)^{1 / 2}}\right)
$$


in terms of the complementary error function. Similarly, the same procedure applied to (4.5) yields

$$
Y_{F}=A_{F} \operatorname{erfc}\left(\frac{\sqrt{L e_{F}}\left(y+y_{F}-\int_{0}^{x} v_{\infty} \mathrm{d} x\right)}{2\left(x-x_{F}\right)^{1 / 2}}\right)
$$

for the small value of the fuel mass fraction near the air boundary. The factors $A_{T}$ and $A_{F}$, the transverse translations $y_{T}$ and $y_{F}$, and the virtual origins $x_{T}$ and $x_{F}$ have values that may be determined by inspection of the numerical results corresponding to the vaporizing mixing layer in a given intermediate range of values of $x$, to provide through (6.3) and (6.4) a universal description of the temperature and fuel fields far from the spray. For instance, for the parametric values considered for heptane in figure $3(a)$, the values $A_{T}=0.2689, A_{F}=1.7482, y_{T}=-0.6209, y_{F}=3.8177$, $x_{T}=0.5321$, and $x_{F}=-2.2994$ are obtained at distances $x$ of order unity, whereas for the parametric values considered for methanol in figure $3(b)$ one obtains the values $A_{T}=0.5184, A_{F}=0.0612, y_{T}=0.8268, y_{F}=1.0665, x_{T}=-0.383$, and $x_{F}=1.2229$. As can be seen, the virtual origins for the temperature and fuel fields are different. In particular, for given boundary conditions the value of $x_{F}$ is seen to be significantly influenced by the latent heat of vaporization $l_{v}$ and specific-heat ratio $c$ of the fuel at hand, with vaporization being facilitated by smaller values of $l_{v}$ and $c$, giving rise to values of the virtual origin $x_{F}$ that are smaller for heptane than they are for methanol. Note that the description given in (6.3) and (6.4) for $x \sim O(1)$ is consistent with the self-similar solution emerging for $x \gg 1$, where $v_{\infty}=V_{\infty} / \sqrt{x}$ with $V_{\infty}$ being a constant, with the temperature and fuel mass fraction taking selfsimilar solutions in terms of $\eta=y / \sqrt{x}$, such that $1-T_{f} \simeq A_{T} \operatorname{erfc}\left[\left(\eta-2 V_{\infty}\right) / 2\right]$ and $Y_{F} \simeq A_{F} \operatorname{erfc}\left[\sqrt{L_{F}}\left(\eta-2 V_{\infty}\right) / 2\right]$ for $\eta \gg 1$.

In the limit of large activation energy $\beta \gg 1$ chemical reaction occurs in a thin layer centred at a transverse location $y_{R}(x) \gg 1$ such that the frozen temperature $T_{f}$ differs from the air temperature by a small amount of order $\beta^{-1}$, a condition that can be expressed in the form

$$
\beta^{-1}=A_{T} \operatorname{erfc}\left(\frac{y_{R}+y_{T}-\int_{0}^{x} v_{\infty} \mathrm{d} x}{2\left(x-x_{T}\right)^{1 / 2}}\right),
$$

which serves to define $y_{R}$ for a given value of $x$. The corresponding fuel mass fraction $Y_{F_{R}}$ at $y=y_{R}$ can be evaluated from (6.4) to give

$$
Y_{F_{R}}=A_{F} \operatorname{erfc}\left(\frac{\sqrt{L e_{F}}\left(y_{R}+y_{F}-\int_{0}^{x} v_{\infty} \mathrm{d} x\right)}{2\left(x-x_{F}\right)^{1 / 2}}\right) \text {. }
$$

Using the asymptotic expansion for large $y$ of the complementary error functions in (6.5) and (6.6) it is easy to see that

$$
y_{R} \sim 2\left(x-x_{T}\right)^{1 / 2} \ln ^{1 / 2}(\beta)
$$


and that

$$
Y_{F_{R}} \sim \beta^{-l_{F}},
$$

where

$$
l_{F}=L e_{F}\left(x-x_{T}\right) /\left(x-x_{F}\right)
$$

is an effective fuel Lewis number. According to (6.8), the amount of fuel available at the reaction layer depends not only on the fuel diffusivity but also on its heating and vaporization characteristics, which determine through $l_{v}$ and $c$ the virtual origins appearing in (6.9). In terms of the estimate (6.8), the criterion (6.1), determining whether sufficient fuel is available for a local thermal runaway to occur, becomes

$$
q \beta^{1-l_{F}} \gg 1 \text {. }
$$

For heptane, the value of $q$ is quite large (i.e. $q=39.5$ when the air temperature is taken to be $1000 \mathrm{~K}$ ) and the values of $c$ and $l_{v}$ are sufficiently small that $x_{F}<x_{T}$, thereby resulting in a small enough value of $l_{F}<L e_{F}$ such that the criterion (6.10) is satisfied under most conditions. The opposite is observed for methanol ignition, because, although the value of $L e_{F}$ is smaller than that of heptane, the associated heat of reaction $q$ is smaller and the values of $c$ and $l_{v}$ are much larger.

For conditions under which the criterion (6.10) is satisfied ignition can be expected to take place as a local thermal explosion centred at $y=y_{R}$ at a given ignition location $x_{I}$. Using the asymptotic expansion for large $y$ of the complementary error functions in (6.5) and (6.6) indicates that the characteristic thickness of the reaction layer scales with $y_{R}^{-1} \ll 1$, so that introduction of the normalized coordinate

$$
\xi=\exp \left(-\frac{y_{R}\left(y-y_{R}\right)}{2\left(x-x_{T}\right)}\right)
$$

simplifies the frozen temperature and fuel mass fractions to

$$
\beta\left(1-T_{f}\right)=\xi \quad \text { and } \quad Y_{F}=Y_{F_{R}} \xi^{l_{F}}
$$

with the modified fuel Lewis number $l_{F}$ appearing as an exponent in the fuel mass fraction. Ignition can be described by writing (4.7) for the rescaled temperature increment $\theta=\beta\left(T-T_{f}\right)$ with fuel consumption neglected, an appropriate simplification when (6.10) holds. The accumulation term is seen to be a factor $y_{R}^{2}$ smaller than the conduction term, so that the problem reduces to a balance between conduction and chemical reaction according to

$$
\frac{\mathrm{d}^{2} \theta}{\mathrm{d} \xi^{2}}=\Delta_{I} \xi^{l_{F}^{-2}} \mathrm{e}^{\theta-\xi}, \quad \theta(0)=\frac{\mathrm{d} \theta}{\mathrm{d} \xi}(\infty)=0,
$$

where

$$
\Delta_{I}=4\left(x-x_{T}\right)^{2} \Delta \beta q Y_{F_{R}} / y_{R}^{2}
$$

is the ignition Damköhler number.

As shown by Liñán \& Williams (1993a), the problem (6.13) has two solutions for a value of $\Delta_{I}$ smaller than a critical value $\Delta_{I, C}$ and no solution for $\Delta_{I}>\Delta_{I, C}$. The dependence of $\Delta_{I, C}$ on $l_{F}$ is shown in figure 8. This figure, together with (6.5), (6.6), (6.9) and (6.14) provide a coupled system of equations that determine the ignition location $x_{i g n}$ as well as the corresponding values of $y_{R}, Y_{F_{R}}, l_{F}$, and $\Delta_{I, C}$. Note that, because of the quasi-steady approximation adopted in deriving (6.13), the 


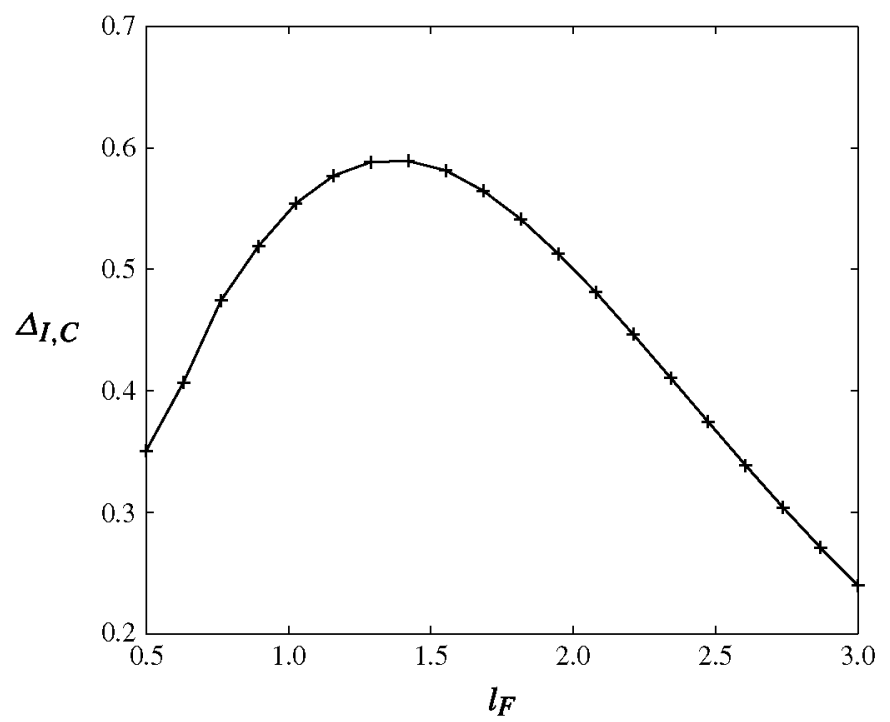

FIGURE 8 . The variation of the critical Damköhler number for ignition $\Delta_{I, C}$ with $l_{F}$ as obtained from integrations of (6.13).
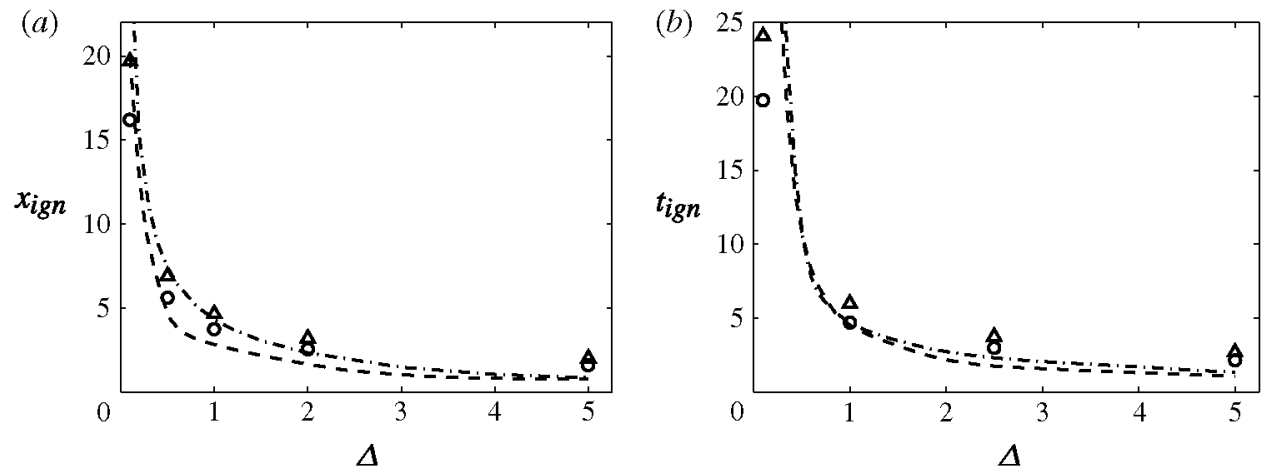

FIGURE 9. The variation with the Damköhler number $\Delta$ of the $(a)$ ignition distance $x_{i g n}$ for the coflow mixing layer with $u_{s}=0.8$ and $(b)$ ignition time $t_{i g n}$ for the unstrained mixing layer (i.e. $u_{s}=1.0$ ) as obtained for a heptane spray from numerical integration of the original problem (4.2)-(4.21) (symbols) and from the asymptotic prediction obtained by evaluating (6.5), (6.6), (6.9), and (6.14) (lines), for sprays carried by air (circles and dashed lines) and inert gas (triangles and dot-dashed lines).

asymptotic development necessarily contains errors that are of order $y_{R}^{2} \sim 1 / \ln \beta$, to be kept in mind when assessing the comparison shown in figure $9(a)$ between the predictions of the asymptotic analysis and those of the numerical integrations. As can be seen, despite its inherent logarithmic errors, the level of accuracy displayed by the asymptotic prediction is satisfactory.

Straightforward replacement of $x$ with $t$ in (6.2)-(6.14) provides formulae for the evaluation of the ignition-delay time $t_{i g n}$ of unstrained spray flamelets. The chemically frozen evolution prior to ignition needs to be considered to determine the parameters 
entering (6.5), (6.6), (6.9) and (6.14), including the values of $A_{T}, A_{F}, y_{T}$, and $y_{F}$, along with the virtual origins $t_{T}$ and $t_{F}$ that replace $x_{T}$ and $x_{F}$ in the temporal description. The results of the asymptotic analysis so obtained are compared with the numerical simulations in figure $9(b)$, which shows a level of agreement similar to that displayed in figure $9(a)$ for the coflow mixing layer. The closeness of the values of $x_{i g n}$ and $t_{i g n}$ in the two figures is indicative of the similarity of the two problems, with $x_{i g n}$ being slightly less than $t_{i g n}$ because of the somewhat lower velocity in the spray streams.

\section{Distributions of mixture fraction and scalar dissipation rate in the igniting mixing layer}

Mixture fractions and magnitudes of their gradients, called scalar-dissipation rates or, more briefly, scalar dissipation (based on their appearance in conservation equations for averages) are widely used in computations, analyses, and modelling of turbulent combustion (Peters 2000). It is important to recognize that there are many different definitions of mixture-fraction fields. They are introduced most readily for two-stream problems, that is, for problems in which inlet streams are of only two distinct types, typically one containing fuel and the other oxidizer, all fuel streams having identical compositions, and similarly for all oxidizer streams. The most basic definition of a mixture-fraction field is the fraction of mass of the material at any given position and time that originated in one of the two streams (by convention, the fuel stream). Such a definition can even be applied to partially premixed two-stream gaseous combustion problems, although that generally is not done because it requires treating the same chemical species in different streams as different species, augmenting the set of differential conservation equations by equations containing coefficients of selfdiffusion, and extending the augmented system to a similar treatment of all combustion intermediaries and products.

The same fuel-stream-related definition can also be introduced for multiphase systems, such as the present spray-combustion problems, including the partially premixed case in which the droplets are transported in an air stream. Just as for mixture-fraction definitions based on element mass fractions, any such definition results in a conserved scalar, in the sense that source terms in principle cannot appear in any differential equation for such a mixture fraction, in this case simply as a consequence of mass conservation. In spray-combustion problems, however, such a definition encounters the severe difficulty that fundamentally a differential conservation equation for this mixture-fraction field does not exist. Any such equation would, for example, be different in the liquid phase and in the gas phase. Moreover, this mixture fraction would, in general, be discontinuous at liquid-gas interfaces. Even when it can be measured in principle, its direct numerical simulation would be prohibitively complex. Unless modelling hypotheses are introduced at the outset, it is not a useful definition for spray-combustion analyses. Nevertheless, some progress in modelling spray combustion has been made with this approach (Bilger 2011; Santasu, Lakshmisha \& Bilger 2011).

\subsection{The gas-phase mixture fraction}

An alternative definition of a mixture fraction in spray combustion that is more useful in many respects is one that focuses only on the gas phase. This makes good sense because a mixture fraction would be irrelevant in the liquid phase. This mixture fraction and its corresponding conservation equation can be derived, as was done for gaseous combustion by Liñán \& Williams (1993b), pp. 143-145, by considering a 
linear combination of the conservation equations for oxygen and fuel vapour that is free from the chemical source term (Arrieta-Sanagustín et al. 2013). The essential results can be illustrated by taking the Lewis number of the fuel to be unity, which simplifies the equations. With this simplification, adding (4.6) and (4.5) times $S$ leads, after use is made of (4.3), to the conservation equation

$$
\frac{\partial(\rho u Z)}{\partial x}+\frac{\partial(\rho v Z)}{\partial y}=\frac{\partial}{\partial y}\left(T^{\sigma} \frac{\partial Z}{\partial y}\right)+\alpha n \dot{m}_{d},
$$

for the gas-phase mixture-fraction variable

$$
Z=\frac{S Y_{F}-\hat{Y}_{O}+1}{S+1}
$$

commonly employed in combustion. Equation (7.1) clearly demonstrates that, for spray combustion, this mixture-fraction variable fundamentally is not a conserved scalar, in the sense that it has a source associated with droplet vaporization.

While (7.1) is sufficiently accurate for methanol, for which the associated Lewis number is close to unity, it is inaccurate for most of the other spray fuels, which typically have vapours with mass diffusivities much smaller than the thermal diffusivity, resulting in Lewis numbers significantly larger than unity, as seen for heptane in table 1 . When a non-unity fuel Lewis number is taken into account, the linear combination corresponding to that leading to (7.1) gives instead the equation

$$
\frac{\partial(\rho u Z)}{\partial x}+\frac{\partial(\rho v Z)}{\partial y}=\frac{S / L e_{F}+1}{S+1} \frac{\partial}{\partial y}\left(T^{\sigma} \frac{\partial \tilde{Z}}{\partial y}\right)+\alpha n \dot{m}_{d},
$$

involving a diffusion-weighted mixture-fraction variable $\tilde{Z}=\left(S Y_{F} / L e_{F}-\hat{Y}_{O}+\right.$ 1) $/\left(S / L e_{F}+1\right)$ besides the unweighted mixture-fraction variable defined in (7.2). An accompanying coupling function free from chemistry effects can be derived for the temperature by combining linearly (4.6) with (4.7) to give

$$
\frac{\partial(\rho u H)}{\partial x}+\frac{\partial(\rho v H)}{\partial y}=\frac{\partial}{\partial y}\left(T^{\sigma} \frac{\partial H}{\partial y}\right)-\alpha n\left[\dot{m}_{d}\left(q / S+l_{v}-T_{B}+1\right)+\dot{q}_{d}\right],
$$

for the total enthalpy

$$
H=T-1+\left(\hat{Y}_{O}-1\right) q / S,
$$

which, just as the mixture fraction, is not a conserved scalar as a result of the presence of source terms, involving in this case the heat transferred from the gas to the droplets. In writing (7.4) the droplet temperature $T_{d}$ has been replaced by the boiling temperature $T_{B}$, in agreement with the simplified description defined in (4.16) and (4.17). If the Lewis number of the oxidizer were not unity, then a diffusionweighted $H$, analogous to $\tilde{Z}$, would also have to be introduced.

For numerical integrations one may in general replace two of the three equations (4.5)-(4.7) by (7.3) and (7.4). The analysis simplifies further in the Burke-Schumann limit of infinitely fast reaction rate, which arises when the reaction time is much smaller than the characteristic fluid mechanical times in the problem. Under those conditions, the chemical reaction is confined to a thin flame, which becomes a sheet when the limit of infinitely fast combustion is considered, separating a region free from oxidizer from a region free from fuel vapour. The flame is located where both $Y_{F}$ and $\hat{Y}_{O}$ are simultaneously zero, corresponding to the stoichiometric 

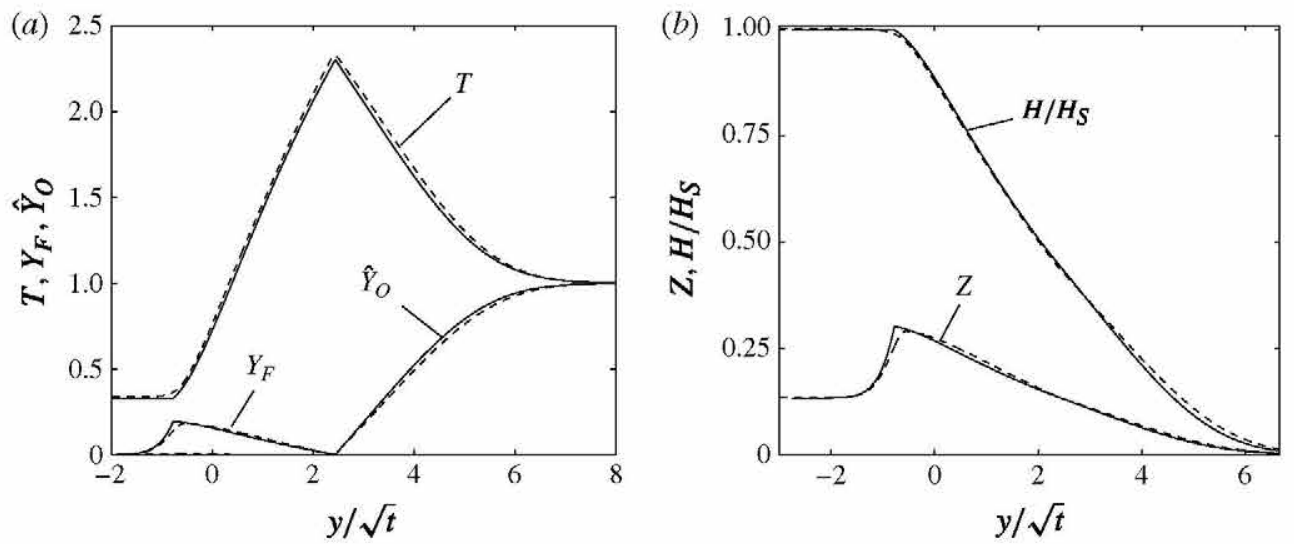

FIGURE 10. Profiles of $Z, H, \hat{Y}_{O}, Y_{F}$, and $T$ across the unstrained unsteady mixing layer obtained from integration of the coupling-function equations (7.1) and (7.4) as described in the Appendix (solid curves) and from extending to $t=65$ the numerical integrations of the methanol spray of figure $7(a-c)$ (dashed curves).

values $Z=Z_{s t}=1 /(S+1)$ and $\tilde{Z}=\tilde{Z}_{s t}=1 /\left(S / L e_{F}+1\right)$ of the ordinary and diffusionweighted mixture fractions. The condition that the reactants cannot coexist can be used to derive the equations

$$
\begin{aligned}
& Z \geqslant Z_{s t}: \hat{Y}_{O}=0, \quad Y_{F}=\frac{Z-Z_{s t}}{1-Z_{s t}}=\frac{\tilde{Z}-\tilde{Z}_{s t}}{1-\tilde{Z}_{s t}}, \quad T=1+H+q / S, \\
& Z \leqslant Z_{s t}: Y_{F}=0, \quad \hat{Y}_{O}=1-\frac{Z}{Z_{s t}}=1-\frac{\tilde{Z}}{\tilde{Z}_{s t}}, \quad T=1+H+\left(Z / Z_{s t}\right)(q / S),
\end{aligned}
$$

linking the values of $Z, \tilde{Z}$ and $H$ everywhere in the flow field. This enables the integration of (7.3) and (7.4) to be performed and provides the mass fractions of reactants and the temperature in terms of the coupling-function variables.

As previously mentioned, the limit of infinitely fast reaction rate applies, for instance, to the description of non-premixed sprays in coflow mixing layers far downstream from the ignition point, the case shown in the heptane computation of figure 4. Correspondingly, infinitely fast reaction can also be used to describe spray diffusion flames in unsteady, unstrained spray mixing layers long after ignition. A sample computation of the latter is shown in figure 10 . In the computation, which is described in detail in the Appendix, the parameters are taken to be those of figure $7(a-c)$, except for the fuel Lewis number, which is assumed to be unity, thereby simplifying the description by enabling (7.1) to be used instead of (7.3) for the computation of the mixture fraction. The resulting self-similar solution therefore corresponds, approximately, to that approached by the unsteady methanol flamelet of figure $7(a-c)$ for $t \gg t_{i g n}$. This is verified by including in the plots of figure 10 the methanol profiles of $\hat{Y}_{O}, Y_{F}$ and $T$ determined numerically at $t=65$ and also the accompanying functions $Z$ and $H$ computed by using these profiles to evaluate (7.2) and (7.5). The degree of agreement of the finite-rate-chemistry results with the coupling-function results is seen to be quite satisfactory. This simple example therefore serves to illustrate the predictive capability of the mixture-fraction formalism in 


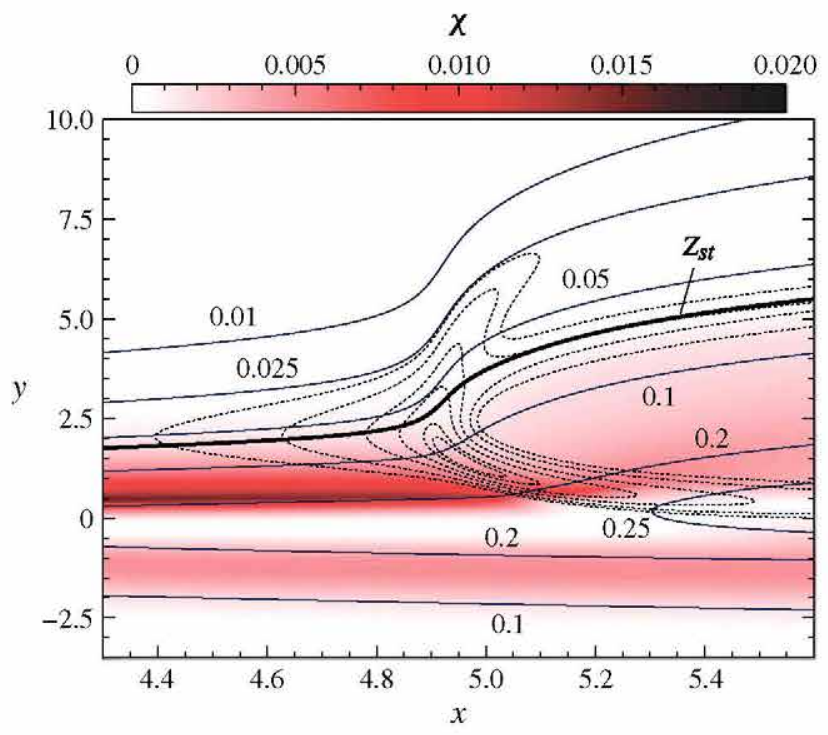

FIGURE 11. (Colour online) Contours of the mixture fraction $Z$ (solid lines) overlaid on reaction-rate isocontours (dotted lines) and filled contours of the scalar dissipation rate $\chi$ (colour online), for ignition in a strained mixing layer of heptane carried by inert gas, in the same conditions as in figure $3(a)$.

computations of spray diffusion flames with fast chemistry, when appropriate account is taken of the spray-source terms affecting $Z$ and $H$ in their conservation equations.

Gas-phase mixture fractions analogous to (7.2) have been used widely in turbulent spray flames for analyses of direct numerical simulation results (Reveillon \& Versvich 2000; Reveillon \& Vervisch 2005; Luo et al. 2011) and for flamelet combustion modelling with finite-rate chemistry (Baba \& Kurose 2008; Franzelli, Fiorina \& Darabiha 2013).

Often in numerical approaches an approximate differential equation for $Z$, free from source terms, is assumed and solved, along with other conservation equations, to determine the $Z$ field (Pitsch \& Peters 1998; Knudsen \& Pitsch 2010; Shashank 2011), sometimes accompanied by a progress-variable equation to account for partial premixing (Peters 2000; Knudsen \& Pitsch 2010). Such a source-free conservation equation is more attractive computationally than (7.1) (or (7.3)) because the latter requires closure modelling for the spray-vaporization term in both the filtered equation and the associated conservation equation for the subgrid variance. The results, however, may miss essential physics in spray flames, associated with the source terms. Results of our computations can be used to test the accuracy of the approximations for $Z$ that have been introduced in the literature. The following observations emphasize computed characteristics of the $Z$ fields and their scalar-dissipation counterparts that bear on such tests and that were not initially expected.

\subsection{Multivalued mixture-fraction fields}

The mixture fraction is not always a single-valued function of the transverse coordinate in the mixing layer of the spray. This is clearly shown in figure 10 for the downstream methanol diffusion flame and also in figures 11 and 12 for the flow field near the ignition kernel. In particular, figure 11 shows the contours of $Z$ calculated 

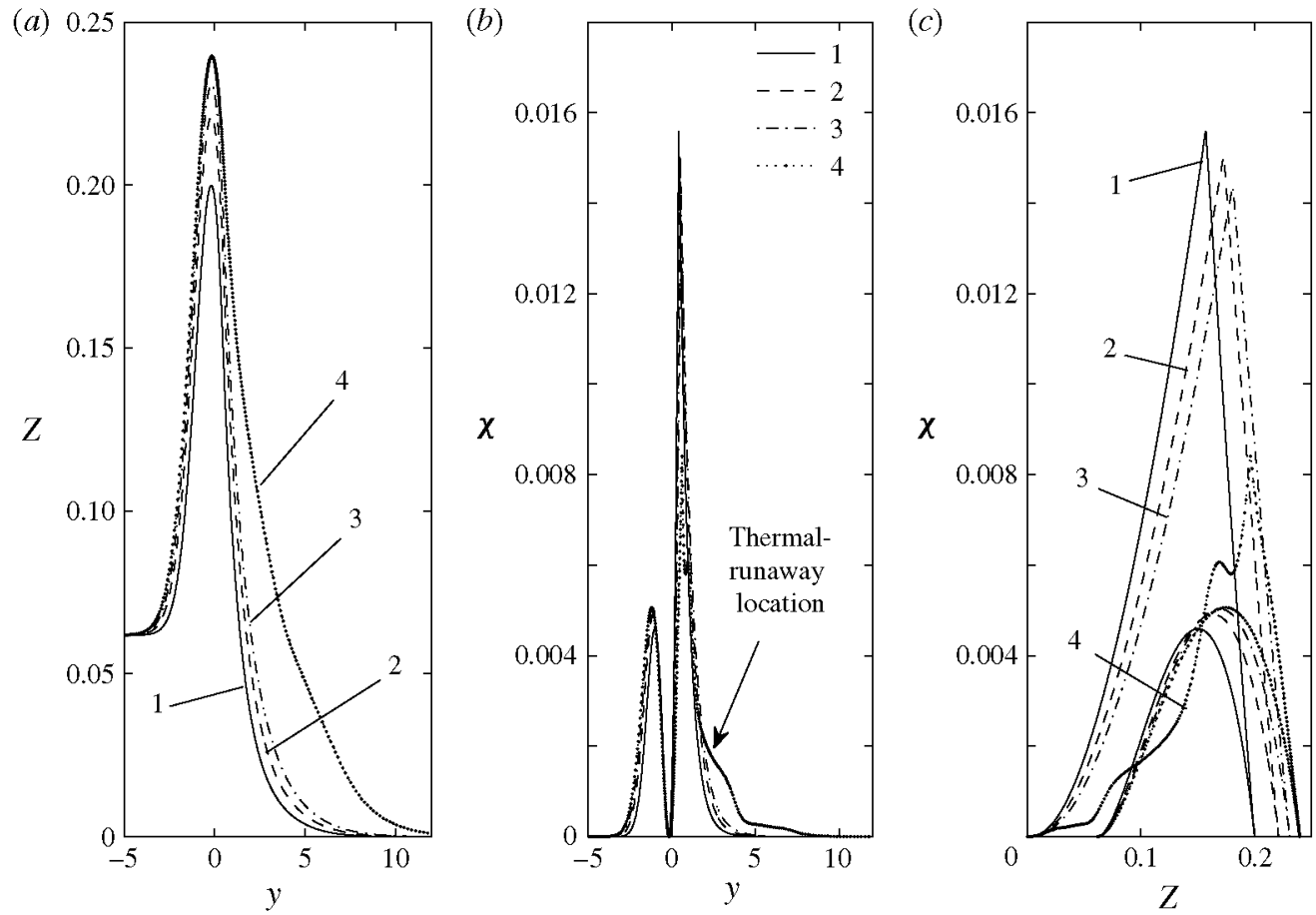

FIGURE 12. Temporal evolutions of (a) mixture fraction $Z$, (b) dimensionless scalar dissipation rate $\chi$ in physical space, and $(c)$ dimensionless scalar dissipation rate $\chi$ in mixture-fraction space, for an unstrained mixing layer of a heptane spray carried by an inert gas, in the same conditions as in figure $6(a-c)$. The time instants $1,2,3$ and 4 correspond, respectively, to $t=4.5,5.5,6.0$, and 6.5 .

by using (7.2) for the strained mixing layer of figure 3(a), which corresponds to a heptane spray carried by an inert gas. It is observed that $Z$ varies from small, near-stoichiometric conditions in the spray stream $(y \rightarrow-\infty)$ because of the absence of fuel vapour there, reaches a maximum within the vaporization zone, and then decays to $Z=0$ in the hot-air stream $(y \rightarrow+\infty)$. In this case since none of the fuel is vaporized in the feed stream of the spray, $Z=Z_{s t}=1 /(1+S)$ there according to (7.2). For sprays carried by air, the mixture fraction actually decays to $Z=0$ in the spray stream, thus producing a bell-shaped spatial distribution. Additionally, in the combustion of sprays the dimensionless scalar dissipation rate $\chi$ non-dimensionalized with $\rho_{A} / t_{v}$, which is defined here as

$$
\chi=T^{\sigma}\left(\frac{\partial Z}{\partial y}\right)^{2}
$$

(to leading order in $1 / P e$ for strained mixing layers), reaches zero within the vaporization region $y \simeq 0$ as shown by the coloured contours of figure 11. This is because of the bell-shaped $Z$ distribution, in which the maximum of $Z$ clearly enforces $\chi=0$ at that location. It is also observed in figure 11 that ignition by thermal runaway occurs in regions of low scalar dissipation rate immediately above the vaporization region, where fuel vapour is available in a high-temperature region in a manner made 
possible by (6.1). The low $\chi$ promoting ignition is quite different from the nearby low $\chi$ in the vaporizing layer.

Similar trends are observed in figure 12 for the temporal evolution of $Z$ and $\chi$ in the same unstrained mixing layer of the heptane spray carried by inert gas that was shown in figure $6(a-c)$. In particular, the ignition by thermal runaway occurs at $y \sim 3$ in a region of low scalar dissipation rate. Two sharp peaks in the scalar dissipation rate occur on both sides of the vaporization layer, which here sits near the centreline of the mixing layer and occupies spatial scales of order unity, the value $\chi=0$ being reached within the vaporization layer as in figure 11. The resulting dependence of $\chi$ on $Z$ is shown in figure $12(c)$. By contrast, in laminar gaseous diffusion flames the scalar dissipation rate is a bell-shaped curve with $\chi=0$ only at the two free streams $Z=0$ and $Z=1$, quite different from figure 12(c) (Peters 2000). This provides a one-to-one transform from physical space to $Z$ space, which is lost in the combustion of sprays. The dual-peak $\chi$ profiles (figure 12) are a direct consequence of the bell-shaped $Z$ profiles for the sprays and are unavoidable with the definition (7.2).

The complex behaviour of this mixture fraction may be understood most easily by assuming that the mass diffusivities of all reactants are equal to the thermal diffusivity (unity Lewis numbers) and deriving a transport equation for $Z$ by combining linearly the fuel and oxidizer transport equations (4.5) and (4.6), which gives (7.1). As previously remarked, this now simply and explicitly shows that $Z$ no longer is a conserved scalar. In (7.1), the source term $\dot{m}_{d}$ is non-zero only in regions where fuel vapour is generated, which suggests that $Z$ behaves as a conserved scalar on both sides of the vaporization layer. The source term $\dot{m}_{d}$ becomes localized in space in the sheath-vaporization limit that is approached sufficiently far downstream, as shown in figures 4 and 10. Near the ignition kernel, however, the thickness of the vaporization layer is comparable to the mixing-layer thickness, with the consequence that typically there is no localization of $\dot{m}_{d}$ in mixture-fraction space, as will be illustrated in $\$ 7.4$.

\subsection{Mixture fraction and scalar dissipation in the gaseous region}

Simplifications occur when attention is restricted to the gaseous region far from the vaporization layer, $y \gg 1$. This region is relevant here for analysing the distribution of $Z$ near the flame when $Z_{s t} \ll 1$, and in this region (7.1) becomes

$$
\frac{\partial Z}{\partial x}+v_{\infty} \frac{\partial Z}{\partial y}=\frac{\partial^{2} Z}{\partial y^{2}},
$$

subject to $Z=0$ at $y \rightarrow+\infty$. A procedure for solving (7.8) can be followed here which is similar to that described in $\S 6$ to derive (6.3) and (6.4). The result is

$$
Z=\frac{A_{Z}}{2} \operatorname{erfc}\left(\frac{\left(y+y_{Z}-\int_{0}^{x} v_{\infty} \mathrm{d} x\right)}{2\left(x-x_{Z}\right)^{1 / 2}}\right),
$$

where $A_{Z}$ is a numerical constant, and $y_{Z}$ and $x_{Z}$ are virtual origins for the mixture fraction, the values of which are obtained by numerical integration. The values of $A_{Z}, y_{Z}$, and $x_{Z}$ depend on the parameters appearing in $\S 4$, in particular on the spray parameters in table 1 and on the velocity ratio $u_{S}=U_{S} / U_{A}$ between the spray and the air streams, which is representative of an applied strain rate. From (7.9) the tail of the 
scalar dissipation is obtained as

$$
\chi=\frac{A_{Z}^{2}}{2 \pi\left(x-x_{Z}\right)} \exp \left\{-2\left[\operatorname{erfc}^{-1}\left(2 Z / A_{Z}\right)\right]^{2}\right\}
$$

for $y \gg 1$. For strained mixing layers, the effective strain rate $\partial u /\left.\partial y\right|_{y \gg 1}$ nondimensionalized with $1 / t_{v}$, affects the prefactor $A_{Z}^{2} /\left(x-x_{Z}\right)$ in (7.10). For unstrained mixing layers this prefactor corresponds to the factor $t^{-1}$ in the scalar dissipation rate in purely gaseous flows (see (3.56) in Peters 2000) which describes its decay with time. These considerations show approximate analogies between purely gaseous ignition and spray ignition at low Stokes numbers, up to factors and time translations, in scalar dissipation near the ignition region and in the flame far from the vaporization layer. This is where flamelet modelling of ignition may apply at low Stokes numbers. The results for sprays depend on the spray dynamics, in that the constants $A_{Z}$ and $x_{Z}$, affecting the shape of $\chi$, depend on the spray parameters $\alpha, S t, \ell_{v}, c, W_{F} / W_{A}$, and $T_{B}$.

\subsection{Flamelet equations for the igniting mixing layer}

Ignition in unstrained mixing layers may be addressed in $Z$ space by expressing the conservation equations (4.5)-(4.7) as

$$
\begin{gathered}
\rho \frac{\partial Y_{F}}{\partial t}+\alpha n \dot{m}_{d}\left\{\frac{\partial Y_{F}}{\partial Z}(1-Z)-\left(1-Y_{F}\right)\right\}=\chi \frac{\partial^{2} Y_{F}}{\partial Z^{2}}-\Delta \Omega, \\
\left.\rho \frac{\partial \hat{Y}_{O}}{\partial t}+\alpha n \dot{m}_{d}\left\{\frac{\partial \hat{Y}_{O}}{\partial Z}(1-Z)+\hat{Y}_{O}\right)\right\}=\chi \frac{\partial^{2} \hat{Y}_{O}}{\partial Z^{2}}-S \Delta \Omega, \\
\rho \frac{\partial T}{\partial t}+\alpha n\left\{\dot{q}_{d}+\dot{m}_{d}\left[\ell_{v}+T-T_{d}++(1-Z) \frac{\partial T}{\partial Z}\right]\right\}=\chi \frac{\partial^{2} T}{\partial Z^{2}}+q \Delta \Omega,
\end{gathered}
$$

in which unity Lewis numbers have been assumed and in which the change of variables $x \rightarrow t$ has been performed. Generalizations to remove these restrictions should be evident from the preceding development but are not exhibited here because their greater complexity serves only to obscure the essential conclusions. The presence of the additional terms within curly brackets in (7.11)-(7.13) is due to the vaporization of the spray. The form of (7.11)-(7.13) is independent of the flow configuration to the extent that Z-field curvature effects are negligible. These particular equations represent the flamelet equations for the combustion of equidiffusive monodisperse sprays with finite-rate chemistry at low Stokes number.

Use of flamelet equations for gaseous fuels generally involves modelling of $\chi(Z)$. Equations (7.11)-(7.13) indicate that, for sprays, models for the distributions $n(Z)$, $\dot{m}_{d}(Z), \dot{q}_{d}(Z)$, and $T_{d}(Z)$ also are needed. An example of these distributions is given in figure 13 for the product $n(Z) \dot{m}_{d}(Z)$. The portions of the curves between $Z=Z_{s t}$ and $\chi=0$ should be excluded in flamelet modelling for the case plotted here because they lie within the spray where chemical reactions do not occur. The curves end at the spray boundary, which often is encountered before $Z$ decreases again towards $Z_{s t}$, reaching the region where ignition is most likely. For sufficiently volatile fuels, the additional terms might have little influence on the flamelet modelling in that they may not affect the tail of $\chi$ significantly near $Z=Z_{s t}$, except for the spray corrections shown in (7.6); we have found this to be approximately true by comparing the curves in figure $12(c)$ with $Z$-field computations that ignore these source terms. However, for non-volatile sprays, combustion may occur near or within the spray cloud, and 


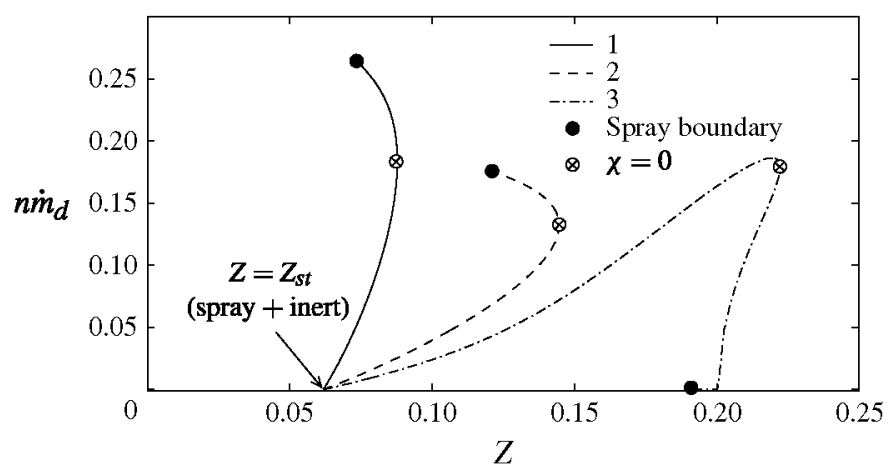

FIGURE 13. Distributions of the dimensionless spray vaporization rate per unit volume $n \dot{m}_{d}$ in $Z$ space for an unstrained mixing layer of heptane carried by inert gas with unity Lewis numbers. The time instants 1,2 and 3 correspond, respectively, to $t=1.1,3.5$ and 5.2.

associated flamelet calculations would then necessarily involve modelling of these terms, which surely would be an ambitious task.

\section{Conclusions}

Ignition of non-premixed and partially premixed spray flames in laminar mixing layers separating a monodisperse spray stream from a hot-air stream have been investigated both numerically and theoretically. This laminar configuration is reasoned to be representative of the reactive flow found in the mixing layers wrapped around the cores of the large convective eddies that develop in turbulent spray mixing layers, where the low-Stokes-number droplets tend to accumulate. Two different ignition modes are encountered depending on the thermochemical properties of the fuel, namely: (i) a thermal-runaway mode in which ignition occurs through a rapid temperature increase; and (ii) a more gradual ignition mode in which the small amount of fuel vapour reaching the hot boundary burns in a lean premixed flame that propagates across the mixing layer towards the spray side. The former type of ignition, found when the thermochemical properties of heptane are employed in the integrations, can be anticipated to be the preferred mode of ignition for highly volatile liquid fuels with large values of the heat of combustion, whereas fuels of lower volatility and with smaller chemical heat release will have a more pronounced tendency to ignite in the slow-deflagration-wave mode, which is observed to occur in our numerical integrations when the properties of methanol are employed for the fuel. Differences in fuel volatility were previously suggested to be one of the main reasons for the significant quantitative differences in ignition delay times and minimum ignition energies found in experimental and numerical studies of methanol and heptane sprays (Danis, Namer \& Cernansky 1988; Gutheil 1995). Activation-energy asymptotics is used to derive analytical predictions for ignition distances associated with thermalrunaway events, giving quantitative results in good agreement with the numerical integrations for heptane.

The numerical integrations serve to clarify different aspects of the igniting solution. For non-premixed configurations, in which the spray is carried by an inert gas, ignition eventually leads to the formation of a trailing diffusion flame that sits far on the air side of the mixing layer at a location such that the fuel vapour generated by the vaporizing spray and the oxygen of the air stream meet in stoichiometric proportions. 
For the partially premixed configurations corresponding to sprays carried by air, depending on the specific boundary conditions considered, the numerical integrations give either solutions including a lean deflagration propagating into the spray followed by a region of distributed reaction or a double-flame configuration including a rich deflagration and a diffusion flame bounding an intermediate oxygen-free region where the droplets vaporize to generate a large fuel-vapour pocket.

The results of the integrations are used to evaluate mixture-fraction and scalar dissipation fields in spray mixing layers. Differences between spray and purely gaseous mixture-fraction-based models of combustion are highlighted, such as the multivalued spatial dependence of the mixture fraction in sprays, which has neither been identified previously nor considered in flamelet modelling. Also emphasized are the complexity of the resulting scalar dissipation rate and the possible need for modelling additional vaporization terms arising in the augmented flamelet equations for spray combustion. It would be of interest to investigate introduction of the spray distribution function to generalize these results from monodisperse to polydisperse sprays.

\section{Acknowledgements}

This work was supported by the Spanish MCINN through project \# CSD201000010 and by the US AFOSR Grant \# FA9550-12-1-0138. J.U. was supported by the 2011-2012 Postdoctoral Fellowship for Excellence in Research, Ibercaja Foundation (Zaragoza, Spain). This investigation was completed during a two-month stay of D.M.R. at the Center for Turbulence Research at Stanford.

\section{Appendix}

The mixture fraction $Z$ and the total enthalpy $H$ defined in (7.2) and (7.5) can be used to describe the solution that appears in non-premixed spray mixing layers far downstream from the ignition point, i.e. at distances $x \gg x_{i g n}$. As shown in figure 4 for heptane, the flow field structure includes a droplet-vaporization layer, whose characteristic thickness is of order unity with the scales selected here, embedded in a thick mixing layer of characteristic thickness $\sqrt{x}$. The mixture fraction $Z$ peaks within the vaporization layer, where the temperature differs from the boiling value by a small amount $T-T_{B} \sim 1 / \sqrt{x} \ll 1$. No vaporization takes place outside the vaporization layer, because $a=0$ on the air side and $T \leqslant T_{B}$ on the spray side. The flame lies on the oxidizer side of the mixing layer at a location to be determined as part of the integration. The corresponding sheath-vaporization solution, similar to that encountered in previous works (Arrieta-Sanagustín et al. 2011, 2013), can be described by considering the asymptotic limit $x \gg 1$ and introducing the similarity variables of order unity $\eta=y / \sqrt{x}$ and $V=\sqrt{x} v$. Integrating the conservation equations in the vaporization-free streams with appropriate jump conditions at the separating vaporization sheet provides a free-boundary parabolic problem that determines the locations of the vaporization and flame sheets, $\eta_{V}=y_{V} / \sqrt{x}$ and $\eta_{f}=y_{f} / \sqrt{x}$, along the peak value $Z=Z_{V}$ of the mixture fraction at $\eta=\eta_{V}$.

As in the computations for figures 6 and 7 , an equivelocity mixing layer with $T_{S}=T_{B}$ will be considered below. For simplicity, the analysis also assumes that the Lewis number of the fuel is unity, thereby enabling (7.1) to be used in the computation. Under these conditions, the solution for the gas phase in the outer non-vaporizing stream for $\eta>\eta_{V}$ is determined by integration of (4.3), (7.1) and (7.4) 
written in the similarity form

$$
\begin{aligned}
(\rho V)_{\eta}-\eta \rho_{\eta} / 2 & =0, \\
\left(T^{\sigma} Z_{\eta}\right)_{\eta}+\rho(\eta / 2-V) Z_{\eta} & =0, \\
\left(T^{\sigma} H_{\eta}\right)_{\eta}+\rho(\eta / 2-V) H_{\eta} & =0,
\end{aligned}
$$

with the subscript $\eta$ denoting differentiation with respect to this variable. The equations must be supplemented with the equation of state (4.2) and with the couplingfunction relationships (7.6), which enable the computation of $\rho$ and $T$ as functions of $Z$ and $H$. The solution is simpler for $\eta<\eta_{V}$ because with $T=T_{B}$ and $\hat{Y}_{O}=0$ the total enthalpy on the spray side of the vaporization layer remains equal to its spray-side boundary value $H=H_{S}=-\left(q / S+1-T_{B}\right)$. For the development below, it is of interest that in this low-Stokes-number region the velocity of the droplets $v_{d}$ differs by a small amount of order $x^{-1}$ from the gas-phase velocity $v$, as follows from the limiting form of (4.13) for $x \gg 1$. This condition can be used to combine (4.3) with (4.9) to yield $\partial(n / \rho) / \partial x+v \partial(n / \rho) / \partial y=0$, which can be integrated along the trajectories to give

$$
n=\rho / \rho_{S},
$$

where $\rho_{S}=T_{B}^{-1}$ is the known value of the gas-phase density in the unperturbed spray stream.

The integration of (A 1)-(A 3) requires consideration of the jumps of $V, Z_{\eta}$ and $H_{\eta}$ at $\eta=\eta_{V}$, to be determined by investigating the vaporization layer in terms of the translated coordinate $\xi=y-y_{V}=y-\eta_{V} x^{1 / 2}$ and the rescaled coupling functions $\mathscr{H}=\left(H-H_{S}\right) x^{1 / 2}=\left(T-T_{B}\right) x^{1 / 2}$ and $\mathscr{Z}=\left(Z-Z_{V}\right) x^{1 / 2}$. The computation begins by integrating the reduced form (4.9),

$$
-\frac{1}{2} \eta_{V} n_{\xi}+(n V)_{\xi}=0
$$

with the boundary conditions $n-n^{-}=V-V^{-}=0$ as $\xi \rightarrow-\infty$ to give

$$
n\left(V-\frac{\eta_{V}}{2}\right)=n^{-}\left(V^{-}-\frac{\eta_{V}}{2}\right)
$$

In this notation, the subscript $\xi$ is used to indicate differentiation with respect to this variable, and the superscripts + and - denote values on the upper and lower sides of the vaporization layer, respectively. In particular, the boundary value of the number density appearing in (A 6) can be evaluated from (A 4) to give

$$
n^{-}=\frac{\rho_{V}}{\rho_{S}}
$$

where

$$
\rho_{V}=T_{B}^{-1}\left[1-\frac{Z_{V}-Z_{s t}}{1-Z_{s t}}\left(1-\frac{W_{A}}{W_{F}}\right)\right]^{-1}
$$

is the gas density at the vaporization layer, expressed here in terms of the unknown peak mixture fraction $Z_{V}$ by virtue of (4.2) and (7.6). Using (A 6) and (A 7) in writing (4.3) and (4.11) in terms of the rescaled variables yields

$$
V_{\xi}=\frac{\alpha}{\rho_{S}}\left(\frac{\eta_{V}}{2}-V^{-}\right)\left(a^{3}\right)_{\xi}=\frac{\alpha n a T_{B}^{\sigma} \mathscr{H}}{\rho_{V} l_{v}} .
$$


On the other hand, the conservation equations for the coupling functions in the vaporization layer can be written as

$$
-\frac{\mathscr{Z}_{\xi \xi}}{\left(1-Z_{V}\right)}=\frac{\mathscr{H}_{\xi \xi}}{l_{v}}=\frac{\alpha n a \mathscr{H}}{l_{v}} .
$$

Combining (A9) and (A 10) to eliminate the vaporization term and integrating the result across the layer provides the equations

$$
V^{+}-V^{-}=\frac{\alpha}{\rho_{S}}\left(V^{-}-\frac{\eta_{V}}{2}\right)=-\frac{T_{B}^{\sigma}\left(Z_{\eta}^{+}-Z_{\eta}^{-}\right)}{\rho_{V}\left(1-Z_{V}\right)}=\frac{T_{B}^{\sigma} H_{\eta}^{+}}{\rho_{V} l_{v}},
$$

when use is made of the identities $Z_{\eta}=\mathscr{Z}_{\xi}$ and $H_{\eta}=\mathscr{H}_{\xi}$.

Integrating (A 1)-(A 3) for $\eta>\eta_{V}$ with boundary conditions $Z=H=0$ as $\eta \rightarrow \infty$ and $Z-Z_{V}=H-H_{S}=V-V^{+}=0$ at $\eta=\eta_{V}^{+}$and (A 1) and (A 2) with boundary conditions $Z-Z_{s t}=V=0$ as $\eta \rightarrow-\infty$ and $Z-Z_{V}=V-V^{-}=0$ at $\eta=\eta_{V}^{-}$, subject to the jump conditions given in (A 11), provides the profiles of $V, Z$ and $H$ across the mixing layer, along with the values of $\eta_{V}, Z_{V}, V^{+}$and $V^{-}$. The solution obtained with the values $T_{B}=0.34, W_{A} / W_{F}=0.91, Z_{S t}=0.133$ and $H_{S}=3.52$, consistent with the parameters given for methanol in table 1, is shown as solid curves in figure 10.

AggarwaL, S. K. 1998 Review of spray ignition phenomena: present status and future research. Prog. Energy Combust. Sci. 24, 565-600.

AnNamalai, K. \& RyAn, W. 1992 Interactive processes in gasification and combustion. 1. Liquid-drop arrays and clouds. Prog. Energy Combust. Sci. 18, 221-295.

Arrieta-Sanagustín, J., SÁnchez, A. L., Liñán, A. \& Williams, F. A. 2011 Sheath vaporization of a monodisperse fuel-spray jet. J. Fluid Mech. 675, 435-464.

Arrieta-Sanagustín, J., Sánchez, A. L., Liñán, A. \& Williams, F. A. 2013 Couplingfunction formulation for monodisperse spray diffusion flames with infinitely fast chemistry. Fuel Process. Technol. 107, 81-92.

BABA, Y. \& Kurose, R. 2008 Analysis and flamelet modelling for spray combustion. J. Fluid Mech. 612, 45-79.

Bermúdez, A., Ferrín, J. L. \& LiÑán, A. 2007 The modelling of the generation of volatiles, $\left(\mathrm{H}_{2}\right.$ and $\mathrm{CO}$, and their simultaneous diffusion controlled oxidation, in pulverized coal furnaces. Combust. Theor. Model. 11, 949-976.

BILGER, R. W. 2011 A mixture fraction framework for the theory and modelling of droplets and sprays. Combust. Flame 158, 191-202.

Chapman, D. R. 1949 Laminar mixing of a compressible fluid. NACA-TN-1800.

ChIU, H. H. \& LiU, T. M. 1977 Group combustion of liquid droplets. Combust. Sci. Technol. 17, $127-142$.

CiEZKI, H. K. \& Adomeit, G. 1993 Shock-tube investigation of self-ignition of n-heptane-air mixtures under engine relevant conditions. Combust. Flame 93, 421-433.

Correa, S. M. \& SiCHEL, M. 1982 The group combustion of a spherical cloud of monodisperse fuel droplets. Proc. Combust. Inst. 19, 981-991.

Crowe, C., Sommerfeld, M. \& Tsuj, Y. 1998 Multiphase Flows with Droplets and Particles. CRC Press.

Danis, A. M., NAmer, I. \& Cernansky, N. P. 1988 Droplet size and equivalence ratio effects on spark ignition of monodisperse n-heptane and methanol spray. Combust. Flame 74, 285-294.

Faeth, G. M. 1983 Evaporation and combustion of sprays. Prog. Energy Combust. Sci. 9, 1-76.

Fernández-Tarrazo, E., SÁnchez, A. L. \& Williams, F. A. 2013 Hydrogen-air mixing-layer ignition at temperatures below crossover. Combust. Flame 160, 1981-1989.

Franzelli, B., Fiorina, B. \& Darabiha, N. 2013 A tabulated chemistry method for spray combustion. Proc. Combust. Inst. 34, 1659-1666. 
Godsave, G. A. E. 1953 Evaporation and combustion of sprays: the burning of single drops of fuel. Proc. Combust. Inst. 4, 818-830.

GutheIL, E. 1995 Numerical analysis of the autoignition of methanol, ethanol, n-heptane and n-octane sprays with detailed chemistry. Combust. Sci. Technol. 34, 1659-1666.

HARRJE, D. T. 1972 Liquid Propellant Rockets. NASA monograph.

Jenny, P. B., Roekaerts, D. \& Beishuizen, N. 2013 Modelling of turbulent dilute spray combustion. Prog. Energy Combust. Sci. 38, 846-887.

KNUdSEN, E. \& Pitsch, H. 2010 Large eddy simulation of a spray combustor using a multi-regime flamelet approach. In Annual Research Briefs, pp. 337-350. Center for Turbulence Research, NASA Ames/Stanford University.

LABOWSKY, M. \& RoSNER, D. E. 1978 Group combustion of droplets in fuel clouds, I. Quasi-steady predictions. In Evaporation-Combustion of Fuels (ed. J. T. Zung), pp. 63-79. American Chemical Society.

LefebVRe, A. 1998 Gas Turbine Combustion. Taylor and Francis.

LESSEN, M. 1950 On the stability of the laminar free boundary between parallel streams. NACA-R-979.

LI, S. C. 1997 Spray stagnation flames. Prog. Energy Combust. Sci. 23, 303-347.

LiÑán, A. 1985 Theory of droplet vaporization and combustion. In Modélisation des Phénomènes de Combustion (ed. R. Borghi, P. Clavin, A. Liñán, P. Pelcé \& G. I. Sivashinsky), CEA-EDF INRIA 59, pp. 73-103. Editions Eyrolles.

LIÑÁN, A. \& CRESPO, A. 1976 An asymptotic analysis of unsteady diffusion flames for large activation energies. Combust. Sci. Technol. 14, 95-117.

LiÑÁN, A. \& WilliaMs, F. A. $1993 a$ Ignition in an unsteady mixing layer subject to strain and variable pressure. Combust. Flame 95, 31-46.

LiÑán, A. \& Williams, F. A. 1993 b Fundamental Aspects of Combustion. Oxford University Press.

LONGMiRe, E. \& EATON, J. K. 1992 Structure of a particle-laden round jet. J. Fluid. Mech. 236, $217-257$.

Luo, K., Pitsch, H., Pai, M. G. \& Desjardins, O. 2011 Direct numerical simulations and analysis of the three-dimensional n-heptane spray flames in a model swirl combustor. Proc. Combust. Inst. 33, 2143-2152.

Mastorakos, E. 2009 Ignition of turbulent non-premixed flames. Prog. Energy Combust. Sci. 35, 57-97.

MoIn, P. \& APTE, S. V. 2006 Large-eddy simulation of realistic gas turbine combustors. AIAA J. 44, 698-708.

Neophytou, A., Mastorakos, E. \& Cant, R. S. 2012 The internal structure of igniting turbulent sprays as revealed by complex chemistry DNS. Combust. Flame 159, 641-664.

Peters, N. 2000 Turbulent Combustion. Cambridge University Press.

PitsCh, H. \& PETERs, N. 1998 A consistent flamelet formulation for non-premixed combustion considering differential diffusion effects. Combust. Flame 114, 26-40.

REVEILLON, J. \& VERSVICH, L. 2000 Spray vaporization in non-premixed turbulent combustion modelling: a single droplet model. Combust. Flame 121, 75-90.

REVEILLON, J. \& VERVISCH, L. 2005 Analysis of weakly turbulent dilute-spray flames and spray combustion regimes. J. Fluid Mech. 537, 317-347.

SÁnCHEZ, A. L. 1997 Nonpremixed spontaneous ignition in the laminar wake of a thin splitter plate. Phys. Fluids 9, 2032-2044.

Santasu, D., LAKshmisha, K. N. \& Bilger, R. W. 2011 Modelling of nonreacting and reacting turbulent spray jets using a fully stochastic separated flow approach. Combust. Flame 158, 1992-2008.

Shashank, 2011 High-fidelity simulations of reactive liquid-fuel jets, PhD thesis, Stanford University.

SiRignano, W. A. 1983 Fuel droplet vaporization and spray combustion theory. Prog. Energy Combust. Sci. 9, 291-322.

Sirignano, W. A. 2010 Fluid Dynamics and Transport of Droplets and Sprays. Cambridge University Press. 
URZAY, J., PITSCH, H. \& LINAN, A. 2011 Source terms for calculations of vaporizing and burning fuel sprays with non-unity Lewis numbers in gases with temperature-dependent thermal conductivities. In Annual Research Briefs, pp. 199-211. Center for Turbulence Research, NASA Ames/Stanford University.

WANG, Y. \& RUTLAND, C. J. 2007 Direct numerical simulation of ignition in turbulent n-heptane liquid-fuel spray jets. Combust. Flame 149, 353-365.

Williams, F. A. 1985 Combustion Theory, 2nd edn. pp. 446-484. Benjamin Cummings.

YING, H. \& YANG, V. 2009 Dynamics and stability of lean-premixed swirl-stabilized combustion. Prog. Energy Combust. Sci. 35, 293-264. 